\title{
Closed forms and multi-moment maps
}

\author{
Thomas Bruun Madsen and Andrew Swann
}

\begin{abstract}
We extend the notion of multi-moment map to geometries defined by closed forms of arbitrary degree. We give fundamental existence and uniqueness results and discuss a number of essential examples, including geometries related to special holonomy. For forms of degree four, multi-moment maps are guaranteed to exist and are unique when the symmetry group is $(3,4)$-trivial, meaning that the group is connected and the third and fourth Lie algebra Betti numbers vanish. We give a structural description of some classes of $(3,4)$-trivial algebras and provide a number of examples.
\end{abstract}

\section{Contents}

1 Introduction

2 Distinguished differential forms 3

3 Multi-moment maps in general degree 7

3.1 Calculus of multi-vectors . . . . . . . . . . . . 8

3.2 Symmetries and multi-moment maps . . . . . . . . 9 9

4 Example geometries and their multi-moment maps 13

4.1 Multi-phase space. . . . . . . . . . . . . . 14

4.2 Product manifolds . . . . . . . . . . . . . . . . . . 14

4.3 Symplectic manifolds . . . . . . . . . . . . . . . . 15

4.4 HyperKähler manifolds . . . . . . . . . . . . . . . 16

4.5 Holonomy $\operatorname{Spin}(7) \ldots \ldots \ldots$. . . . . . . . 17

$4.6 \quad \mathrm{G}_{2}$-manifolds . . . . . . . . . . . . . . . 20

4.7 PSU(3)-structures . . . . . . . . . . . . . . . . . . 23

4.8 Homogeneous k-plectic manifolds . . . . . . . . 2 24

5 Cohomology of Lie algebras 25

$5.1 \quad(3,4)$-trivial Lie algebras $\ldots \ldots \ldots$

2010 Mathematics Subject Classification: Primary 53C15; Secondary 22E25, 53C29, 53C30, 53C55, 53D20, 70G45. 


\section{Introduction}

The rich and varied field of symplectic geometry is the study of closed nondegenerate two-forms. It has origins in the study of Hamiltonian dynamics and the geometry of phase space. From a mathematical point of view it is natural to try to see how much of this theory may be extended to closed forms of higher degree. A number of authors have already made attempts at generalising the Hamiltonian picture to higher-degree, or multi-, phase spaces, often motivated by the interest in various field theories $[11,12,19,1$, 2]. Indeed string- and $M$-theories with fluxes give a number of geometries equipped with closed differential forms of varying degrees, see [20] for one such example.

The purpose of this article is to study the geometry of closed differential forms in general, with particular emphasis on new techniques that are available in the presence of symmetry. One main tool in the construction of various symplectic manifolds is the Marsden-Weinstein quotient formed by taking quotients of the level sets of a moment map. One important feature of the moment map in symplectic geometry, is that it takes values in a finite-dimensional vector space depending only on the symmetry group and not on the underlying manifold. Previous attempts to extend moment maps to forms of higher degrees, have produced maps taking values in infinite-dimensional spaces of forms over the manifold, see the references above, though [32] provides an interesting alternative. In [29] we introduced a new notion of multi-moment map for geometries with a closed three-form, which shares the above basic property of symplectic moment maps. A thorough study of these new maps was made in [30]. In this paper we will show how this theory extends to forms of arbitrary degree, in large part based on ideas developed in the thesis [28]. Not only do these multimoment maps take values in a finite-dimensional vector space, but there are existence results based on easily satisfied properties of the manifold or its symmetry group. We will thus describe the general theory, give examples of multi-moment reduction of various geometries, particular ones with a closed four-form, and study an algebraic condition on Lie groups that guarantees existence and uniqueness of multi-moment maps for fourform geometries.

One salient feature of symplectic geometry is that the two-form is nondegenerate. What this means for a form of higher degree is less clear and we start the paper in Section 2 by discussing a number of different possibilities. These distinguish a number of geometries that have importance in their own right, for example geometries with exceptional holonomy, but do not lead to any one good constraint, so for the general theory we do not impose such assumptions.

In Section 3 we then introduce the notion of multi-moment map for symmetries of closed geometries of arbitrary degree. In order to facilitate the 
proofs we develop some theory of multi-vectors on manifolds and in particular give an extension of the classical Cartan formula which expresses the Lie derivative of forms in terms of exterior derivatives and contractions. Multi-moment maps are then defined, and existence and uniqueness theorems proved under topological and under algebraic assumptions. Section 4 then gives a number of examples of closed geometries, computes multi-moment maps in a number of cases and discusses the geometries of quotients. Finally, in Section 5 we study the algebraic condition found in Section 3.2 for the existence and uniqueness of multi-moment maps for geometries with a closed four-form. These conditions are expressed as the vanishing of the third and fourth Lie algebra cohomology groups. We show how to exploit the Hochschild-Serre spectral sequence to determine the algebraic structure of a wide class of such Lie algebras and give a number of examples.

Acknowledgements We gratefully acknowledge financial support from CTQM, GEOMAPS and OPALGTOPGEO. AFS is also partially supported by the Danish Council for Independent Research, Natural Sciences, Symmetry Techniques in Differential Geometry and by the Ministry of Science and Innovation, Spain, under Project MTM2008-01386. AFS thanks the organisers of the GESTA meeting 2011 for a stimulating event and the opportunity to present aspects of this material.

\section{Distinguished differential forms}

We will be considering geometries defined by closed differential forms. So as a first question we address the issue of whether there are any algebraically distinguished forms on a vector space. Recall that in symplectic geometry one makes repeated use of the 'non-degeneracy' of the symplectic two-form $\omega$. Algebraically this leads to the fact that a symplectic manifold is of even dimension and then the closure of $\omega$ is used for Darboux's Theorem, that there are local coordinates so that $\omega=d x_{1} \wedge d y_{1}+\cdots+d x_{n} \wedge$ $d y_{n}$. For higher degree forms, the situation is not simple and it is not clear which definition is appropriate. Let us discuss some of the possibilities.

Let $V$ be an $n$-dimensional vector space over $\mathbb{R}$. Write $\Lambda^{*} V^{*}$ for the algebra of forms on $V$.

Definition 2.1. A form $\alpha \in \Lambda^{r} V^{*}$ is said to be fully non-degenerate if

$$
\alpha\left(v_{1}, v_{2}, \ldots, v_{r-1}, \cdot\right)
$$

is non-zero whenever $v_{1}, \ldots, v_{r-1} \in V$ are linearly independent. 


\section{Closed Forms AND MULTI-MOMENT MAPS}

For $r=2$, this is the usual non-degeneracy of a two-form. For any two-form $\alpha$ there is a basis of $V^{*}$ such that

$$
\alpha=e_{1} \wedge e_{2}+\cdots+e_{2 k-1} \wedge e_{2 k}
$$

for some $k \leqslant \frac{1}{2} \operatorname{dim} V$. To see this start with a non-zero vector $X \in V$ and put $e_{2}=\alpha(X, \cdot)$. Choose a vector $Y$ such that $e_{2}(Y)=1$ then choose a one-form $e_{1}$ with $e_{1}(X)=1$ and $e_{2}(Y)=0$. We now have $\alpha^{\prime}=\alpha-e_{1} \wedge e_{2}$ is zero on $X$ and $Y$, and the result follows by induction. We see that $\alpha$ is non-degenerate if and only if $\operatorname{dim} V=2 k$.

For forms of degree 3 , full non-degeneracy already gives much stronger restrictions.

Theorem 2.2. A vector space of dimension $n$ admits a fully non-degenerate of form of degree $r \geqslant 3$ if and only if $r=n$ or the pair $(r, n)$ is either $(3,7)$ or $(4,8)$.

Proof. Choose an inner product $\langle\cdot, \cdot\rangle$ on $V$. A form $\alpha \in \Lambda^{r} V^{*}$ defines a cross-product like operation $V^{r-1} \rightarrow V$ via

$$
\left\langle v_{1} \times v_{2} \times \cdots \times v_{r-1}, w\right\rangle=\alpha\left(v_{1}, v_{2}, \ldots, v_{r-1}, w\right) .
$$

This operation is continuous and has the property that the product $v_{1} \times$ $\cdots \times v_{r-1}$ is orthogonal to each of the $v_{i}$. When $\alpha$ is fully non-degenerate, this product on linearly independent vectors is non-zero. Let $V_{r, n}$ denote the Stiefel manifold consisting of all $r$-tuples $\left(f_{1}, \ldots, f_{r}\right)$ of orthonormal vectors in $\mathbb{R}^{n}$. The map

$$
\left(f_{1}, \ldots, f_{r-1}\right) \mapsto\left(f_{1}, \ldots, f_{r-1}, \frac{f_{1} \times \cdots \times f_{r-1}}{\left\|f_{1} \times \cdots \times f_{r-1}\right\|}\right)
$$

is a cross section of the projection $V_{r, n} \rightarrow V_{r-1, n}$. It is a topological result of Whitehead [38] that such a cross-section exists only in the given cases. An elementary proof for the case of two-fold cross-products, $r=3$, may be found in [31].

For $r=n$, a volume form on $V$ provides a fully non-degenerate form. Examples for the other two cases of this result are given by the three-forms

$$
\phi_{0}=e_{123}+e_{145}+e_{167}+e_{246}-e_{257}-e_{347}-e_{356}
$$

on $\mathbb{R}^{7}$ and the four-form

$$
\begin{aligned}
\Phi_{0}=e_{1234}+e_{1256}+e_{3478}+e_{3456}+e_{1278}+e_{1357}-e_{1368} \\
-e_{2457}+e_{2468}-e_{1458}-e_{1467}-e_{2358}-e_{2367}+e_{5678}
\end{aligned}
$$

on $\mathbb{R}^{8}$. Here $e_{1}, \ldots, e_{n}$ is a basis for $\left(\mathbb{R}^{n}\right)^{*}$ and wedge products have been omitted from the notation, so $e_{123}=e_{1} \wedge e_{2} \wedge e_{3}$, etc. 
The forms $\phi_{0}$ and $\Phi_{0}$ have interesting geometric properties. In particular, if we consider the action of $G L(n, \mathbb{R})$ then the isotropy groups $\{g \in G L(n, \mathbb{R}): g \cdot \alpha=\alpha\}$ are the compact 14-dimensional exceptional Lie group $G_{2}$ for $\alpha=\phi_{0}$ and the compact 21-dimensional group $\operatorname{Spin}(7)$, the simply-connected double cover of $S O(7)$, for $\alpha=\Phi_{0}$, see Bryant [8]. We now see that the dimensions of the orbits of these forms are

$$
\begin{aligned}
& \operatorname{dim}\left(G L(7, \mathbb{R}) \cdot \phi_{0}\right)=49-14=35 \\
& \operatorname{dim}\left(G L(8, \mathbb{R}) \cdot \Phi_{0}\right)=64-21=43
\end{aligned}
$$

The first of these is notable since $\operatorname{dim} \Lambda^{3} \mathbb{R}^{7}=35$, so the orbit of $\phi_{0}$ in $\Lambda^{3} \mathbb{R}^{7}$ is open.

Definition 2.3 (Hitchin [23]). A form $\alpha \in \Lambda^{r} V^{*}$ is stable if the orbit $G L(V) \cdot \alpha$ is open in $\Lambda^{r} V^{*}$.

For general forms the condition of stability provides restrictions on the dimension of $V$.

Proposition 2.4. A vector space of dimension $n$ admits a stable form of degree $r$ if and only if either $r \in\{1,2, n-2, n-1, n\}$ or $r \in\{3, n-3\}$ with $n \in\{6,7,8\}$.

Proof. We give the basic arguments, following Hitchin [23].

The dimension of the orbit $G L(n, \mathbb{R}) \cdot \alpha$ is at most $\operatorname{dim}(G L(n, \mathbb{R}))=n^{2}$. To have a stable form we thus need $n^{2} \geqslant \operatorname{dim} \Lambda^{r} \mathbb{R}^{n}=\left(\begin{array}{l}n \\ r\end{array}\right)$. The binomial coefficient $\left(\begin{array}{l}n \\ r\end{array}\right)$ is a polynomial of degree $r$ in $n$, which for $3 \leqslant r \leqslant n-3$ grows quicker than $n^{2}$. Now for $r<n / 2$, we have $\operatorname{dim} \Lambda^{r} \mathbb{R}^{n}<\operatorname{dim} \Lambda^{r+1} \mathbb{R}^{n}$, so we start by considering the case $r=3$. In this case, we see that

$$
\begin{aligned}
\operatorname{dim} \Lambda^{3} \mathbb{R}^{n}-\operatorname{dim}(G L(n, \mathbb{R})) & =\frac{1}{6} n(n-1)(n-2)-n^{2} \\
& =\frac{1}{6} n((n-9) n+2)
\end{aligned}
$$

so an orbit in $\Lambda^{3} \mathbb{R}^{n}$ can not be open if $n \geqslant 9$. In dimension $n=8$, we have $\operatorname{dim} \Lambda^{3} \mathbb{R}^{8}<64=\operatorname{dim} G L(8, \mathbb{R})$, but $\operatorname{dim} \Lambda^{4} \mathbb{R}^{8}=70>64$, so orbits of four-forms on $\mathbb{R}^{8}$ are never open. This gives the list of possible $r$ and $n$ in the statement.

It remains to show that each case can be realised. For $r=1, n-1, n$, we take $\alpha$ to be any non-zero form of the given degree. For $r=2$, open orbits are realised by forms as in (2.1) with $k=\lfloor n / 2\rfloor$. Taking the Hodge star of such a two-form gives a stable form of degree $n-2$.

Finally, we need to give appropriate three-forms in dimensions 6, 7 and 8; the case for $r=n-3$ will then follow by taking Hodge stars. For dimension $n=6$, one can take $\alpha$ to be the real part of a complex volume form on $\mathbb{R}^{6}=\mathbb{C}^{3}$. We have already seen $\phi_{0}(2.2)$ is stable on $\mathbb{R}^{7}$. Finally 


\section{Closed Forms AND MULTI-MOMENT MAPS}

for $n=8$, one identifies $\mathbb{R}^{8}$ with the Lie algebra $\mathfrak{s u}(3)$. This carries an adinvariant three-form $\alpha(X, Y, Z)=\langle[X, Y], Z\rangle$, which in an appropriate basis is

$$
\begin{aligned}
\rho_{0}=e_{123}+ & \frac{1}{2} e_{1}\left(e_{47}-e_{56}\right)+\frac{1}{2} e_{2}\left(e_{46}+e_{57}\right) \\
& +\frac{1}{2} e_{3}\left(e_{45}-e_{67}\right)+\frac{\sqrt{3}}{2} e_{8}\left(e_{45}+e_{67}\right) .
\end{aligned}
$$

The infinitesimal stabiliser of this form is $\mathfrak{s u}(3)$ and so the orbit of $\rho_{0}$ has dimension $64-8=56=\operatorname{dim} \Lambda^{3} \mathbb{R}^{8}$ and is open.

Note that the connected subgroup of $G L(8, \mathbb{R})$ preserving $\rho_{0}$ is $\operatorname{PSU}(3)$ : the quotient of $S U(3)$ by its centre $\mathbb{Z} / 3$.

So far we have considered two strong conditions on forms and found them to be rather restrictive. There is another condition that is useful more generally.

Definition 2.5. A form $\alpha$ on $V$ is (weakly) non-degenerate if

$$
v\lrcorner \alpha=\alpha(v, \cdot, \ldots, \cdot)
$$

is non-zero for each non-zero $v$.

Any non-zero form $\alpha$ gives rise to a non-degenerate form on the quotient $V / \operatorname{ker} \alpha$ where $\operatorname{ker} \alpha=\{v \in V: v\lrcorner \alpha=0\}$. Conversely a volume form always provides a non-degenerate form on any vector space. For a particular degree of form there can be restrictions on the dimension. For two-forms weak and full non-degeneracy are the same and the space must be even-dimensional. In higher degree we have far fewer restrictions.

Proposition 2.6. A vector space of dimension $n$ admits a non-degenerate form of degree $r$ with $r \geqslant 3$ if and only if $n \geqslant r$ and $n \neq r+1$.

Proof. For $n<r$, we have $\Lambda^{r} V^{*}=\{0\}$, so all $r$-forms are zero and thus degenerate. For $n=r+1$, any form of degree $r$ is the Hodge dual of a one-form and so has the form $\alpha=e_{2} \wedge \cdots \wedge e_{n}$, which is degenerate.

To demonstrate existence of non-degenerate forms in the remaining cases, first consider $r=3$. If $n \geqslant 3$ is odd, let $\omega$ be a non-degenerate two-form on $\mathbb{R}^{n-1}$, then $\alpha=\omega \wedge e_{n}$ is a non-degenerate three-form on $\mathbb{R}^{n}$. If $n \geqslant 6$ is even, then writing $\mathbb{R}^{n}=\mathbb{R}^{3} \oplus \mathbb{R}^{n-3}$ we have a non-degenerate three-form given by $\alpha=e_{123}+\alpha^{\prime}$, where $e_{123}$ is a volume form on $\mathbb{R}^{3}$ and $\alpha^{\prime}$ is non-degenerate on $\mathbb{R}^{n-3}$.

Now for $r>3$, given a non-degenerate form $\alpha^{\prime}$ of degree $r-1$ on $\mathbb{R}^{n-1}$ we have that $\alpha=\alpha^{\prime} \wedge e_{n}$ is non-degenerate of degree $r$ on $\mathbb{R}^{n}$. 


\section{Multi-moment maps in general degree}

The general situation we wish to study is where a symmetry group $G$ acts on a manifold $M$ preserving some closed form.

Definition 3.1. For $r \geqslant 2$, a closed geometry of degree $r$ on a manifold $M$ is choice of a closed differential form $\alpha \in \Omega^{r}(M)$.

Here $\alpha$ closed means $d \alpha=0$ in the exterior algebra. This implies that if $\operatorname{ker} \alpha$ has constant dimension then $\mathcal{D}=\operatorname{ker} \alpha$ is integrable as a distribution. It follows that $\alpha$ induces a weakly non-degenerate closed form on $M / \mathcal{D}$ when this quotient is a manifold. In general we will not make any non-degeneracy assumptions on $\alpha$. However, when needed, the following terminology will be useful.

Definition 3.2 (Baez, Hoffnung and Rogers [1]). A $k$-plectic structure is a closed geometry $(M, \alpha)$ of degree $r=k+1$ with $\alpha$ (weakly) non-degenerate.

It is easy to give a couple of elementary examples. Firstly, if $M=\mathbb{R}^{n}$ is a vector space, then any constant coefficient form $\alpha$ on $M$ is closed, and the discussion of the previous section gives many $k$-plectic examples. Of particular importance are the forms $\phi_{0}(\underline{2.2})$, on $\mathbb{R}^{7}, \Phi_{0}(\underline{2.3})$ and $\rho_{0}(2.4)$, both on $\mathbb{R}^{8}$.

Example 3.3 (Multi-phase space). Given any manifold $N$ we may consider $M=$ $\Lambda^{k} T^{*} N$. This carries a tautological form $\beta \in \Omega^{k}(M)$ given by

$$
\beta_{b}\left(X_{1}, \ldots, X_{k}\right)=b\left(\pi_{*} X_{1}, \ldots, \pi_{*} X_{k}\right),
$$

where $\pi: M=\Lambda^{k} T^{*} N \rightarrow N$ is the bundle projection. Defining

$$
\alpha=d \beta,
$$

we get a closed $(k+1)$-form on $M$ which turns out to be non-degenerate so $(M, \alpha)$ is $k$-plectic. To see this, choose local coordinates $q^{1}, \ldots, q^{n}$ on $U \subseteq N$ and note that $d q^{I}=d q^{i_{1}} \wedge \cdots \wedge d q^{i_{r-1}}$ gives a basis for each fibre of $\Lambda^{k} T^{*} U \subset M$. Let $p_{I}$ be the corresponding fibre coordinates, then

$$
\beta=\sum_{I} p_{I} d q^{I}, \quad \alpha=\sum_{I} d p_{I} \wedge d q^{I},
$$

and non-degeneracy of $\alpha$ is manifest. This is the higher degree generalisation of the usual symplectic structure on phase space $T^{*} N$. It is the central example in mechanics and field theory based approaches to the geometry of closed forms.

In order to build multi-moment maps we need to construct closed oneforms out of the group action and the closed $r$-form $\alpha$. This involves contracting $\alpha$ with linear combinations of $r-1$ vector fields. Here it is convenient to use the notion of multi-vectors. 


\section{Closed Forms AND MULTI-MOMENT MAPS}

\subsection{Calculus of multi-vectors}

Recall that a multi-vector $p$ of degree $s$ on $M$ is a sum $p=\sum_{i=1}^{k} q_{i}$ of simple multi-vectors of the form

$$
q=X_{1} \wedge X_{2} \wedge \cdots \wedge X_{s}
$$

with $X_{j}$ smooth vector fields on $M$. We will use

$$
\mathfrak{X}^{s}(M)=\Gamma\left(\Lambda^{s} T M\right)
$$

to denote the space of degree $s$ multi-vectors on $M$. This is dual to the space $\Omega^{s}(M)$ of differential forms of the same degree. We write $\lrcorner$ for the partial evaluation map $\mathfrak{X}^{s}(M) \times \Omega^{r}(M) \rightarrow \Omega^{r-s}(M)$,

$$
(q\lrcorner \beta)\left(Y_{1}, \ldots, Y_{r-s}\right)=\beta\left(X_{1}, X_{2}, \ldots, X_{s}, Y_{1}, \ldots, Y_{r-s}\right) .
$$

When we consider symmetries, we will have use for a generalisation of Cartan's formula $\left.\left.\mathcal{L}_{X} \alpha=d(X\lrcorner \alpha\right)+X\right\lrcorner d \alpha$ for the Lie derivative. To this end note that one may regard $\mathfrak{X}(M)$ as a vector space over $\mathbb{R}$ and form the exterior powers $\Lambda_{\mathbb{R}}^{s} \mathfrak{X}(M)$. These spaces are larger than $\mathfrak{X}^{\mathcal{S}}(M)$ which is equal to the exterior product of $\mathfrak{X}(M)$ over $C^{\infty}(M)$. There is a natural $\mathbb{R}$-linear projection $\Lambda_{\mathbb{R}}^{s} \mathfrak{X}(M) \rightarrow \mathfrak{X}^{\mathcal{S}}(M)$ given on decomposable elements by

$$
Q=X_{1} \curlywedge \cdots \curlywedge X_{s} \mapsto q=X_{1} \wedge \cdots \wedge X_{s}
$$

where $\curlywedge$ denotes the wedge product over $\mathbb{R}$. For a such a $Q$, we write

$$
Q_{\wedge i}=(-1)^{i-1} X_{1} \curlywedge \cdots \curlywedge \widehat{X}_{i} \curlywedge \cdots \curlywedge X_{S}
$$

and $Q_{\wedge i j}=\left(Q_{\wedge i}\right)_{\wedge j}$. We define

$$
\begin{aligned}
& \left.(\lrcorner \mathcal{L})_{Q} \alpha=\sum_{i=1}^{s} Q_{\wedge i}\right\lrcorner \mathcal{L}_{X_{i}} \alpha \quad \text { and } \\
& L(Q)=\sum_{1 \leqslant i<j \leqslant s}\left[X_{i}, X_{j}\right] \curlywedge Q_{\wedge i j},
\end{aligned}
$$

and extend $\mathbb{R}$-linearly to $\Lambda_{\mathbb{R}}^{s} \mathfrak{X}(M)$.

Lemma 3.4 (Extended Cartan Formula). For $\alpha \in \Omega^{r}(M)$ and $p \in \mathfrak{X}^{s}(M)$, we have

$$
\left.\left.\left.p\lrcorner d \alpha-(-1)^{s} d(p\lrcorner \alpha\right)=(\lrcorner \mathcal{L}\right)_{P} \alpha-L(P)\right\lrcorner \alpha
$$

for any $P \in \Lambda_{\mathbb{R}}^{s} \mathfrak{X}(M)$ projecting to $p$.

Proof. The left-hand side is independent of the presentation of $p$ and both sides are $\mathbb{R}$-linear, so it is enough to prove the corresponding formula for 
a decomposable $Q$ projecting to $q$ as in (3.2). Note that when $s=r+1$ we have one of the standard formulae for the exterior derivative:

$$
(d \alpha)(q)=(\lrcorner \mathcal{L})_{Q} \alpha-\alpha(L(Q)) .
$$

For general $s \leqslant r+1$, write $Q^{\prime}=Y_{1} \curlywedge \cdots \curlywedge Y_{t}$ with $s+t=r+1$. Note that we always have $Q\lrcorner \beta=q\lrcorner \beta$ for any form $\beta$. Now we compute, using (3.5), (3.3) and (3.4),

$$
\begin{aligned}
& \left.(q\lrcorner d \alpha)\left(q^{\prime}\right)=d \alpha\left(q \wedge q^{\prime}\right)=(\lrcorner \mathcal{L}\right)_{Q \curlywedge Q^{\prime}} \alpha-\alpha\left(L\left(Q \curlywedge Q^{\prime}\right)\right) \\
& =\sum_{i=1}^{s}\left(\mathcal{L}_{X_{i}} \alpha\right)\left(Q_{\wedge i} \curlywedge Q^{\prime}\right)+(-1)^{s} \sum_{j=1}^{t}\left(\mathcal{L}_{Y_{j}} \alpha\right)\left(Q \curlywedge Q_{\wedge j}^{\prime}\right) \\
& \quad-\alpha\left(L(Q) \curlywedge Q^{\prime}\right)-(-1)^{s} \alpha\left(Q \curlywedge L\left(Q^{\prime}\right)\right) \\
& \quad-(-1)^{s} \sum_{i=1}^{s} \sum_{j=1}^{t} \alpha\left(\left[X_{i}, Y_{j}\right] \curlywedge Q_{\wedge i} \curlywedge Q_{\wedge j}^{\prime}\right) \\
& \left.\left.=((\lrcorner \mathcal{L})_{Q} \alpha\right)\left(Q^{\prime}\right)+(-1)^{s} \sum_{j=1}^{t} \mathcal{L}_{Y_{j}}(Q\lrcorner \alpha\right)\left(Q_{\wedge j}^{\prime}\right)-(-1)^{s} \sum_{j=1}^{t} \alpha\left(\mathcal{L}_{Y_{j}} Q \curlywedge Q_{\wedge j}^{\prime}\right) \\
& \left.\quad-(L(Q)\lrcorner \alpha)\left(Q^{\prime}\right)-(-1)^{s}(Q\lrcorner \alpha\right)\left(L\left(Q^{\prime}\right)\right)+(-1)^{s} \sum_{j=1}^{t} \alpha\left(\mathcal{L}_{Y_{j}} Q \curlywedge Q_{\wedge j}^{\prime}\right) \\
& \left.\left.\left.=((\lrcorner \mathcal{L})_{Q} \alpha+(-1)^{s} d(q\lrcorner \alpha\right)-L(Q)\right\lrcorner \alpha\right)\left(q^{\prime}\right),
\end{aligned}
$$

which gives the claimed result.

\subsection{Symmetries and multi-moment maps}

Let $(M, \alpha)$ be a manifold with an $r$-form $\alpha$ not necessarily closed.

Definition 3.5. A group of symmetries of $(M, \alpha)$ is a connected Lie group $G$ acting on $M$ preserving $\alpha$.

Infinitesimally this means that

$$
\mathcal{L}_{X} \alpha=0 \quad \text { for all } X \in \mathfrak{g},
$$

where $\mathfrak{g}$ is the Lie algebra of $G$ and we write $X$ for the vector field generated by $X$.

Example 3.6 (Multi-phase space). Suppose $M=\Lambda^{k} T^{*} N$ with the canonical $k$ plectic form $\alpha$ of Example 3.3. Then any diffeomorphism $\phi$ of the base $N$ induces a symmetry $\psi$ of $(M, \alpha)$ covering $\phi$, namely take $\psi=\left(\phi^{*}\right)^{-1}$. In this way, any group $G$ of diffeomorphisms of $N$ lifts a group of symmetries of $(M, \alpha)$. 


\section{Closed Forms AND MULTI-MOMENT MAPS}

The map sending an element $X$ of $\mathfrak{g}$ to the vector field $X$ on $M$ generated by $X$ is $\mathbb{R}$-linear. So we may extend this to associate to each $p \in \Lambda^{s} \mathfrak{g}$ a unique multi-vector $p \in \mathfrak{X}^{S}(M)$. For a decomposable $q=X_{1} \wedge X_{2} \wedge \cdots \wedge$ $\mathrm{X}_{s}$, the corresponding multi-vector is exactly the $q$ given in equation (3.1). When $G$ preserves $\alpha$, we have (\lrcorner $\mathcal{L})_{p} \alpha=0$ for each $p \in \Lambda^{s} \mathfrak{g}$, so the extended Cartan formula reads

$$
\left.\left.p\lrcorner d \alpha-(-1)^{s} d(p\lrcorner \alpha\right)=-L(p)\right\lrcorner \alpha \quad \text { for all } p \in \Lambda^{s} \mathfrak{g},
$$

where $L(p)$ is understood to be the multi-vector corresponding to $L(\mathrm{p}) \in$ $\Lambda^{s-1} \mathfrak{g}$ which is defined as in (3.4) but using the Lie bracket of $\mathfrak{g}$. In particular, when $\alpha$ is closed, we see that $p\lrcorner \alpha$ is closed whenever $L(p)=0$.

Definition 3.7. The $k$ th Lie kernel of $\mathfrak{g}$ is the $\mathfrak{g}$-module

$$
\mathcal{P}_{\mathfrak{g}, k}=\operatorname{ker}\left(L: \Lambda^{k} \mathfrak{g} \rightarrow \Lambda^{k-1} \mathfrak{g}\right) .
$$

If $G$ acts a group of symmetries for a closed geometry of degree $r$ we may write

$$
\mathcal{P}_{\mathfrak{g}}=\mathcal{P}_{\mathfrak{g}, r-1}
$$

for the corresponding Lie kernel of $\mathfrak{g}$.

Since each $\operatorname{Ad}_{g}, g \in G$, is a Lie algebra automorphism of $\mathfrak{g}$, we see that $\mathcal{P}_{\mathfrak{g}}$ is a $G$-module.

If $G$ is a Abelian, then $\mathcal{P}_{\mathfrak{g}, k}=\Lambda^{k} \mathfrak{g}$. For any $\mathfrak{g}$, we have $\mathcal{P}_{\mathfrak{g}, 1}=\mathfrak{g}$.

Example 3.8. For $G=S U(2)$, let $\mathrm{X}_{1}, \mathrm{X}_{2}, \mathrm{X}_{3}$ be a basis of $\mathfrak{s u}(2)$ satisfying $\left[\mathrm{X}_{1}, \mathrm{X}_{2}\right]=-2 \mathrm{X}_{3}$, etc. The map $L: \Lambda^{3} \mathfrak{s u}(2) \rightarrow \Lambda^{2} \mathfrak{s u}(2)$ is given by the cyclic sum $L\left(\mathrm{X}_{1} \wedge \mathrm{X}_{2} \wedge \mathrm{X}_{3}\right)=\mathfrak{S}_{1,2,3}\left[\mathrm{X}_{1}, \mathrm{X}_{2}\right] \wedge \mathrm{X}_{3}=-2 \sum_{i=1}^{3} \mathrm{X}_{i} \wedge \mathrm{X}_{i}=0$, so this $L$ is identically zero. Thus $\mathcal{P}_{\mathfrak{s u}(2), 2}=\Lambda^{3} \mathfrak{s u}(2)^{*} \cong \mathbb{R}$. On the other hand, $L: \Lambda^{2} \mathfrak{s u}(2) \rightarrow \mathfrak{s u}(2)$ is an isomorphism and $\mathcal{P}_{\mathfrak{s u}(2), 1}=\{0\}$.

More generally, if $\mathfrak{g}$ is a simple Lie group of compact type, and $\mathrm{X}_{1}, \ldots, \mathrm{X}_{n}$ is a basis for $\mathfrak{g}$ consisting of unit length vectors for the Killing form, then one has that the element $\sum_{i, j=1}^{n} \mathrm{X}_{i} \wedge \mathrm{X}_{j} \wedge\left[\mathrm{X}_{i}, \mathrm{X}_{j}\right]$ lies in $\mathcal{P}_{\mathfrak{g}, 2}$. This element corresponds to the standard representative $\gamma(X, Y, Z)=\langle[X, Y], Z\rangle$ of the third cohomology group $H^{3}(\mathfrak{g}) \cong \mathbb{R}$. For $\mathfrak{g}$ simple, $L: \Lambda^{2} \mathfrak{g} \rightarrow \mathfrak{g}$ is onto so we have $\mathcal{P}_{\mathfrak{g}, 1} \cong \Lambda^{2} \mathfrak{g} / \mathfrak{g}$, which is a non-zero irreducible $G$-module when $\operatorname{dim} G>3$, cf. Wolf [41].

Now suppose that we have a closed geometry $(M, \alpha)$ and that $G$ is a group of symmetries. Then for $\mathrm{p}$ in $\mathcal{P}_{\mathfrak{g}}=\mathcal{P}_{\mathfrak{g}, r-1} \leqslant \Lambda^{r-1} \mathfrak{g}$ we have $\left.p\right\lrcorner \alpha \in$ $\Omega^{1}(M)$ and

$$
d(p\lrcorner \alpha)=0,
$$


by Lemma 3.4. Thus $p\lrcorner \alpha$ is a closed one-form and locally the derivative of a function $v_{\mathrm{p}}$. Letting $\mathrm{p}$ vary over $\mathcal{P}_{\mathfrak{g}}$, we obtain a family of functions that may be combined into a local map $v: M \rightarrow \mathcal{P}_{\mathfrak{g}}^{*}$ by setting $\langle v, \mathrm{p}\rangle=v_{\mathrm{p}}$. This motivates the following definition:

Definition 3.9. Let $G$ be a symmetry group for a closed geometry $(M, \alpha)$. A multi-moment map for this action is an equivariant map $v: M \rightarrow \mathcal{P}_{\mathfrak{g}}^{*}$ satisfying

$$
d\langle v, \mathrm{p}\rangle=p\lrcorner \alpha
$$

for all $\mathrm{p} \in \mathcal{P}_{\mathfrak{g}}$.

For $\alpha$ a symplectic form, this is exactly the usual notion of moment map, since $\mathcal{P}_{\mathfrak{g}}=\mathcal{P}_{\mathfrak{g}, 1}=\mathfrak{g}$. Concrete examples of multi-moment maps will be given in Section 4 .

The obstructions to constructing a multi-moment map are quite weak. To start with if $b_{1}(M)=0$ then we can always find global functions $v_{\mathrm{p}}$ with $\left.d v_{\mathrm{p}}=p\right\lrcorner \alpha$. Using averaging arguments we have the following topological existence result.

Theorem 3.10. Let $(M, \alpha)$ be a closed geometry with $G$ acting as a group of symmetries. Suppose $b_{1}(M)=0$. Then there exists a multi-moment map $v: M \rightarrow \mathcal{P}_{\mathfrak{g}}^{*}$ if either

(i) $G$ is compact, or

(ii) $M$ is compact and orientable, and $G$ preserves a volume form on $M$.

Proof. The proofs given in [30] for the case of $\alpha$ a three-form carry directly over to this general situation. In the first case, one averages over $G$; in the second, one averages over $M$.

A second useful existence result occurs when $\alpha$ is exact in a good way.

Proposition 3.11. Suppose $G$ is a group of symmetries of a closed geometry $(M, \alpha)$ of degree $r$. If there exists $a G$-invariant form $\beta$ with $\alpha=d \beta$, then

$$
\langle v, \mathrm{p}\rangle=(-1)^{r-1} \beta(p) \quad \text { for } \mathrm{p} \in \mathcal{P}_{\mathfrak{g}}
$$

defines a multi-moment map for the action of $G$.

Proof. Invariance of $\beta$ implies that $v$ is equivariant, so we just need to verify equation (3.7). However, Lemma 3.4 gives

$$
\left.\left.\left.d\langle v, \mathbf{p}\rangle=(-1)^{r-1} d(p\lrcorner \beta\right)=p\right\lrcorner d \beta=p\right\lrcorner \alpha,
$$

since $L(\mathrm{p})=0$ and $\beta$ is invariant. 


\section{Closed Forms AND MULTI-MOMENT MAPS}

Finally there is a purely algebraic existence and uniqueness result depending only on the Betti numbers of $\mathfrak{g}$. The dual of the map $L: \Lambda^{k+1} \mathfrak{g} \rightarrow$ $\Lambda^{k} \mathfrak{g}$ is essentially the differential

$$
\begin{gathered}
d: \Lambda^{k} \mathfrak{g}^{*} \rightarrow \Lambda^{k+1} \mathfrak{g}^{*} \\
(d \gamma)\left(\mathrm{X}_{1}, \mathrm{X}_{2}, \ldots, \mathrm{X}_{k}\right)=-\gamma\left(L\left(\mathrm{X}_{1} \wedge \mathrm{X}_{2} \wedge \cdots \wedge \mathrm{X}_{k}\right)\right) .
\end{gathered}
$$

The Jacobi identity implies $L \circ L=0$ and so $d \circ d=0$. Thus we have the Lie algebra homology $H_{*}(\mathfrak{g})$ of $\mathfrak{g}$ defined by the complex $\left(\Lambda^{*} \mathfrak{g}, L\right)$ and the Lie algebra cohomology $H^{*}(\mathfrak{g})$ defined by $\left(\Lambda^{*} \mathfrak{g}^{*}, d\right)$. In particular,

$$
H^{k}(\mathfrak{g})=\frac{\operatorname{ker}\left(d: \Lambda^{k} \mathfrak{g}^{*} \rightarrow \Lambda^{k+1} \mathfrak{g}^{*}\right)}{\operatorname{im}\left(d: \Lambda^{k-1} \mathfrak{g}^{*} \rightarrow \Lambda^{k} \mathfrak{g}^{*}\right)}=\frac{Z^{k}(\mathfrak{g})}{B^{k}(\mathfrak{g})}
$$

the quotient of the space $Z^{k}(\mathfrak{g})=$ ker $d$ of cycles by the space of boundaries $B^{k}(\mathfrak{g})=\operatorname{im} d$, and we write

$$
b_{k}(\mathfrak{g})=\operatorname{dim} H^{k}(\mathfrak{g})
$$

for the kth Betti number of $\mathfrak{g}$.

Our algebraic existence and uniqueness criteria are expressed in terms of vanishing of certain Betti numbers. It is therefore useful to introduce the following terminology.

Definition 3.12. A connected Lie group $G$ or its Lie algebra $\mathfrak{g}$ is (cohomologically) $\left(k_{1}, k_{2}, \ldots, k_{\ell}\right)$-trivial if the Betti numbers $b_{k}(\mathfrak{g})$ vanish for $k=$ $k_{1}, k_{2}, \ldots, k_{\ell}$.

We will discuss these type of conditions in some detail in Section 5 , however let us note that a simple Lie algebra is always $(1,2)$-trivial, but has $b_{3}$ non-zero. Indeed looking up the Poincaré polynomials of the compact simple Lie algebras reveals the following:

Proposition 3.13 (Madsen [28]). Every compact simple Lie algebra not isomorphic to $\mathfrak{s u}(n), n \geqslant 3$, is $(1,2,4,5,6)$-trivial.

Returning to multi-moment maps the algebraic existence and uniqueness result is:

Theorem 3.14. Suppose $(M, \alpha)$ is a closed geometry of degree $r$ and $G$ is a group of symmetries. If $G$ is $(r-1, r)$-trivial then there exists a unique multi-moment map v: $M \rightarrow \mathcal{P}_{\mathfrak{g}}^{*}$. If $G$ is just $(r-1)$-trivial, then $v$ is unique whenever it exists.

Proof. The proof builds on the following observation. Taking the dual of the exact sequence

$$
0 \longrightarrow \mathcal{P}_{\mathfrak{g}, k} \stackrel{\iota}{\longrightarrow} \Lambda^{k} \mathfrak{g} \stackrel{L}{\longrightarrow} \Lambda^{k-1} \mathfrak{g}
$$


we obtain the sequence

$$
\Lambda^{k-1} \mathfrak{g}^{*} \stackrel{d}{\longrightarrow} \Lambda^{k} \mathfrak{g}^{*} \stackrel{\pi}{\longrightarrow} \mathcal{P}_{\mathfrak{g}, k}^{*} \longrightarrow 0 .
$$

From this one sees that $\mathcal{P}_{\mathfrak{g}, k}^{*} \cong \Lambda^{k} \mathfrak{g}^{*} / B^{k}(\mathfrak{g})$ and so the exterior derivative $d: \Lambda^{k} \mathfrak{g}^{*} \rightarrow \Lambda^{k+1} \mathfrak{g}^{*}$ induces a well-defined linear map $d_{\mathcal{P}}: \mathcal{P}_{\mathfrak{g}}^{*} \rightarrow \Lambda^{k+1}(\mathfrak{g})$ via $d_{\mathcal{P}} a=d b$ where $a=b+B^{k}(\mathfrak{g}) \in \mathcal{P}_{\mathfrak{g}, k}^{*} \cong \Lambda^{k} \mathfrak{g}^{*} / B^{k}(\mathfrak{g})$. We now see that $d_{\mathcal{P}}$ is injective if and only if $b_{k}(\mathfrak{g})=0$ and that the image of $d_{\mathcal{P}}$ is $B^{k+1}(\mathfrak{g})$.

Now let us consider the situation of the Theorem. The action of $G$ on $M$ defines a map

$$
\begin{gathered}
\Psi: M \rightarrow Z^{r}(\mathfrak{g}), \\
\langle\Psi(x), \mathrm{p}\rangle=(-1)^{r} \alpha(p)_{x}
\end{gathered}
$$

for all $\mathrm{p} \in \Lambda^{r} \mathfrak{g}$ and $x \in M$. To see that the image lies in $Z^{r}(\mathfrak{g}) \leqslant \Lambda^{r} \mathfrak{g}^{*}$, use (3.6) for the invariant closed form $\alpha$ to get

$$
\left.\langle d(\Psi(x)), \mathrm{q}\rangle=\langle\Psi(x), L(\mathbf{q})\rangle=(-1)^{r} \alpha(L(q))_{x}=(-1)^{r+1}(q\lrcorner d \alpha\right)_{x}=0,
$$

for each $\mathrm{q} \in \Lambda^{r+1} \mathfrak{g}$.

Now if $b_{r}(\mathfrak{g})=0$, then $Z^{r}(\mathfrak{g})=B^{r}(\mathfrak{g})=\operatorname{im} d_{\mathcal{P}}$, so we may find for each $x \in M$ a $v_{x} \in \mathcal{P}_{\mathfrak{g}}^{*}$ with $d_{\mathcal{P}}\left(v_{x}\right)=\Psi(x)$. If $b_{r-1}(\mathfrak{g})=0$, the map $d_{\mathcal{P}}$ is injective, so there is a unique choice of $v_{x}$ for each $x$. It follows that $v$ is equivariant.

Suppose we have an equivariant map $v: M \rightarrow \mathcal{P}_{\mathfrak{g}}^{*}$ with $d_{\mathcal{P}} v=\Psi$ of equation (3.10) and that $b_{r-1}(\mathfrak{g})=0$. We claim that $v$ is a multi-moment map. The important fact here is that $b_{r-1}(\mathfrak{g})=0$ says ker $d=\operatorname{im} d$ in $\Lambda^{r-1} \mathfrak{g}^{*}$ which dually means that $\operatorname{im} L=\operatorname{ker} L \operatorname{in} \Lambda^{r-1} \mathfrak{g}$. However, $\operatorname{ker} L=\mathcal{P}_{\mathfrak{g}}$ so $L: \Lambda^{r} \mathfrak{g} \rightarrow \Lambda^{r-1} \mathfrak{g}$ maps on to the Lie kernel $\mathcal{P}_{\mathfrak{g}}$. We may now compute, for $\mathrm{p}=-L(\mathrm{q}) \in \mathcal{P}_{\mathfrak{g}}$

$$
\begin{aligned}
d\langle v, \mathrm{p}\rangle & =-d\langle v, L(\mathrm{q})\rangle=d\left\langle d_{\mathcal{P}}(v), \mathrm{q}\right\rangle \\
& \left.=d\langle\Psi, \mathbf{q}\rangle=(-1)^{r} d(q\lrcorner \alpha\right) \\
& =L(q)\lrcorner \alpha=p\lrcorner \alpha,
\end{aligned}
$$

by (3.6). Thus $v$ is indeed a multi-moment map.

\section{Example geometries and their multi-moment maps}

Having introduced the general theory of multi-moment maps we will now look at a number of concrete examples. For many examples the main focus will be on closed geometries of degree 4, but we will also consider other cases. When relevant we will also discuss the use of multi-moment maps to describe reductions of certain geometries. 


\section{Closed Forms AND MULTI-MOMENT MAPS}

Definition 4.1. Suppose $v: M \rightarrow \mathcal{P}_{\mathfrak{g}}^{*}$ is a multi-moment map. Then for each $t \in \mathcal{P}_{\mathfrak{g}}^{*}$ fixed by the $G$-action, the reduction of $M$ at level $t$ is

$$
M / / v, t G=v^{-1}(t) / G \text {. }
$$

We set $M / /{ }_{v} G=M / /{ }_{v, 0} G$ for the reduction at level 0 .

This makes sense, since $v$ is $G$-equivariant so the $G$-action preserves $v^{-1}(t)$ whenever $t$ is fixed by $G$. This notion of reduction corresponds to the usual Marsden-Weinstein quotient in symplectic geometry. However because the structure of forms of higher degree is so varied, the type of geometry obtained on the quotient is often of a different character to the geometry on $M$. Also, one usually has to impose assumptions, such as freeness of the action of $G$ and regularity of the value $t$, in order to obtain smooth quotients.

\subsection{Multi-phase space}

This is $M=\Lambda^{r-1} T^{*} N$ with the canonical $r$-form $\alpha$ of Example 3.3. If $G$ is any group of diffeomorphisms of $N$, then as noted in Example 3.6, this induces an action of $G$ on $M$ preserving $\alpha$. However, in this case we have $\alpha$ is equivariantly exact: the canonical form $\beta$ is also $G$-invariant and satisfies $d \beta=\alpha$. By Proposition 3.11, there is a multi-moment map $v$ given by $\langle v, \mathrm{p}\rangle=(-1)^{r-1} \beta(p)$.

A concrete example is provided by taking $N=\mathbb{R}^{4}$. If we consider the closed geometry of degree 4 on $M=\Lambda^{3} T^{*} N \cong T N \cong \mathbb{R}^{8}$, we have $\alpha$ and $\beta$ given by the cyclic sums

$$
\alpha=\underset{1,2,3,4}{\mathfrak{S}} d p^{1} \wedge d q^{2} \wedge d q^{3} \wedge d q^{4}, \quad \beta=\underset{1,2,3,4}{\mathfrak{S}} p^{1} d q^{2} \wedge d q^{3} \wedge d q^{4} .
$$

If $G=\mathbb{R}^{4}$ acts by translations on $N=\mathbb{R}^{4}$ then

$$
v\left(\frac{\partial}{\partial q^{1}} \wedge \frac{\partial}{\partial q^{2}} \wedge \frac{\partial}{\partial q^{3}}\right)=p^{4}, \quad \text { etc. }
$$

and $v$ is simply projection on to the fibres of $T \mathbb{R}^{4} \rightarrow \mathbb{R}^{4}$.

\subsection{Product manifolds}

Let $\left(N, \alpha^{\prime}\right)$ be a $(k-1)$-plectic manifold. Consider $M=S^{1} \times N$ and write $\theta$ for the standard one-form on the $S^{1}$-factor. Then as in the proof of Proposition 2.6, we have that $\alpha=\theta \wedge \alpha^{\prime}$ is a $k$-plectic form on $M$.

If $H$ is a group of symmetries of $\left(N, \alpha^{\prime}\right)$, then $G=S^{1} \times H$ is a group of symmetries of $(M, \alpha)$, where the $S^{1}$-factor of $G$ acts non-trivially on just the $S^{1}$-factor of $M$ preserving $\theta$. 
Suppose $v^{\prime}: N \rightarrow \mathcal{P}_{\mathfrak{h}, k-1}^{*}$ is a multi-moment map for the action of $H$ on $N$. Writing $\mathfrak{g}=\mathbb{R} T \oplus \mathfrak{h}$, we have that

$$
\Lambda^{m} \mathfrak{g}=\left(\mathrm{\top} \wedge \Lambda^{m-1} \mathfrak{h}\right) \oplus \Lambda^{m} \mathfrak{h}
$$

Since $T$ commutes with $\mathfrak{h}$, the map $L: \Lambda^{k} \mathfrak{g} \rightarrow \Lambda^{k-1} \mathfrak{g}$ preserves the splittings (4.1) and we conclude that

$$
\mathcal{P}_{\mathfrak{g}, k} \cong\left(\mathrm{T} \wedge \mathcal{P}_{\mathfrak{h}, k-1}\right) \oplus \mathcal{P}_{\mathfrak{h}, k}
$$

As $\left.0=\mathcal{L}_{T} \theta=d(T\lrcorner \theta\right)$, we may scale T by a constant so that $\theta(T)=1$. Let $\vartheta$ denote the element of $\mathfrak{g}^{*}$ that annihilates $\mathfrak{h}$ and has $\vartheta(T)=1$. We claim that

$$
v=\vartheta \wedge v^{\prime}
$$

is a multi-moment map for the action of $G$ on $M$. Firstly, $v$ is a map to $\mathbb{R} \vartheta \wedge \mathcal{P}_{\mathfrak{h}, k-1}^{*} \subset \mathcal{P}_{\mathfrak{g}, k}^{*}$ and it is equivariant for the action of $G=S^{1} \times H$. Secondly, for $\mathrm{p} \in \mathcal{P}_{\mathfrak{g}, k}$, we have $\mathrm{p}=\mathrm{T} \wedge \mathrm{p}^{\prime}+\mathrm{q}$ with $\mathrm{p}^{\prime} \in \mathcal{P}_{\mathfrak{h}, k-1}$ and $\mathrm{q} \in \mathcal{P}_{\mathfrak{h}, k}$. Now $v$ is zero on $q$ and

$$
\left.\left.\left.d\langle v, \mathbf{p}\rangle=d\left\langle\vartheta \wedge v^{\prime}, \mathbf{T} \wedge \mathbf{p}^{\prime}\right\rangle=d\left\langle v^{\prime}, \mathbf{p}^{\prime}\right\rangle=p^{\prime}\right\lrcorner \alpha^{\prime}=\left(T \wedge p^{\prime}\right)\right\lrcorner\left(\theta \wedge \alpha^{\prime}\right)=p\right\lrcorner \alpha .
$$

So $v$ satisfies (3.7) and is a multi-moment map.

Starting with $(N, \omega)$ a symplectic manifold with a Hamiltonian action of $H$, iteration of the above construction produces a $k$-plectic structure on $M=T^{k-1} \times N$ together with a multi-moment map for the action of $G=$ $T^{k-1} \times H$.

\subsection{Symplectic manifolds}

If $\omega \in \Omega^{2}(M)$ is an ordinary symplectic form, then each power $\omega^{k} \in$ $\Omega^{2 k}(M)$ with $2 k \leqslant \operatorname{dim} M$ is $(2 k-1)$-plectic. In particular, we may consider the four-form $\alpha=\omega \wedge \omega=\omega^{2}$ as a 3-plectic form on $M$. Let us take $\operatorname{dim} M \geqslant 6$ and assume that $M$ is simply-connected.

If $X$ is a vector field preserving $\alpha$, then we have $0=\mathcal{L}_{X} \alpha=2 \omega \wedge \mathcal{L}_{X} \omega$. But the map $\omega \wedge \cdot: \Lambda^{2} T^{*} M \rightarrow \Lambda^{4} T^{*} M$ is injective when $\operatorname{dim} M \geqslant 6$, so we have $\mathcal{L}_{X} \omega=0$ and $X$ also preserves $\omega$. Thus symmetries of $\left(M, \omega^{2}\right)$ are nothing but symplectomorphisms of $(M, \omega)$.

Let us first consider actions of Abelian groups. Suppose $G=\mathbb{R}^{3}$ acts generated by vector fields $X_{1}, X_{2}$ and $X_{3}$. Then by the extended Cartan formula (Lemma 3.4), we have $\left.\left.d\left(\omega\left(X_{i}, X_{j}\right)\right)=d\left(\left(X_{i} \wedge X_{j}\right)\right\lrcorner \omega\right)=-\left[X_{i}, X_{j}\right]\right\lrcorner \omega=$ 0 , showing that $\omega\left(X_{i}, X_{j}\right)$ is constant. Taking constant linear combinations of our vector fields we may therefore assume that $\omega\left(X_{i}, X_{3}\right)=0$, for $i=1,2$, and that $\omega\left(X_{1}, X_{2}\right)=\delta \in\{0,1\}$. 


\section{Closed Forms AND MULTi-MOMENT MAPS}

Now a multi-moment map $v: M \rightarrow \mathcal{P}_{\mathfrak{g}}^{*}=\Lambda^{3} \mathfrak{g}^{*} \cong \mathbb{R}$ has differential

$$
\begin{aligned}
d v & \left.\left.=\left(X_{1} \wedge X_{2} \wedge X_{3}\right)\right\lrcorner \alpha=\left(X_{1} \wedge X_{2} \wedge X_{3}\right)\right\lrcorner \omega^{2} \\
& \left.=\underset{1,2,3}{\mathfrak{S}} \omega\left(X_{1}, X_{2}\right) \omega\left(X_{3}, \cdot\right)=\delta X_{3}\right\lrcorner \omega .
\end{aligned}
$$

Thus if $\delta=0$, i.e. the orbits of the $G$-action are isotropic, then $v$ is constant. Fixing now $\delta=1$, we note that if $\mu_{i}$ is a symplectic moment map for $X_{i}$, then $X_{2} \mu_{1}=d \mu_{1}\left(X_{2}\right)=\omega\left(X_{1}, X_{2}\right)=\delta=1$. Thus in this case $X_{1}$ and $X_{2}$ do not Poisson commute and there is no symplectic moment map for the action of the whole of $G$. However, $v=\mu_{3}$ is a multi-moment map for the action of $G$.

A second case is given by considering $G=S U(2)$ with generators $X_{i} \in$ $\mathfrak{s u}(2)$ as in Example 3.8. Since $G$ is compact and $b_{1}(M)=0$, there is a symplectic moment map $\mu=\left(\mu_{1}, \mu_{2}, \mu_{3}\right): M \rightarrow \mathfrak{s u}(2)^{*} \cong \mathbb{R}^{3}$ satisfying $L_{X_{1}} \mu_{2}=-2 \mu_{3}$, etc. As above we find that $L_{X_{1}} \mu_{2}=d \mu_{2}\left(X_{1}\right)=-\omega\left(X_{1}, X_{2}\right)$. For a multi-moment map $v: M \rightarrow \mathcal{P}_{\mathfrak{s u}(2), 2} \cong \mathbb{R}$, equation (4.2) gives

$$
\left.d v=\left(X_{1} \wedge X_{2} \wedge X_{3}\right)\right\lrcorner \alpha=2 \sum_{i=1}^{3} \mu_{i} d \mu_{i}=d\|\mu\|^{2} .
$$

Thus a multi-moment map is $v=\|\mu\|^{2}$. It is unique up to the addition of a constant.

The quotient $M / / v S U(2)=v^{-1}(0) / S U(2)$ is nothing other than the symplectic quotient of $M$ by $S U(2)$, and thus inherits both a symplectic form $\omega^{\prime}$ and a closed four-form $\alpha^{\prime}=\left(\omega^{\prime}\right)^{2}$.

For $t>0$, the geometry of the reduction $M / / v, t S U(2)=v^{-1}(t) / S U(2)$ is more complicated. Part of the reason for this is that even in the good case when $S U(2)$ acts freely on $M_{t}$, there is no canonical choice of connection form for the $S U(2)$-bundle $M_{t} \rightarrow M / / v, t S U(2)$. This problem is remedied in geometries that come equipped with a metric.

\subsection{HyperKähler manifolds}

A variant of the construction considered above arises in the setting of quaternionic geometry. A quaternion-Hermitian manifold $Q$ is a $4 n$-dimensional Riemannian manifold with a rank three subbundle $\mathcal{G} \subset \operatorname{End}(T Q)$ which is locally trivialised by anti-commuting almost complex structures $I, J$ and $K$ that satisfy $K=I J$. In addition the Riemannian metric $g$ must be compatible with $\mathcal{G}$, meaning $g(\mathcal{I} X, \mathcal{I} Y)$ for each $X, Y \in T_{x} Q$ and $\mathcal{I} \in \mathcal{G}_{x}$; in particular $\omega_{I}=g(I \cdot, \cdot)$, etc., are locally defined non-degenerate two-forms. A quaternion-Hermitian manifold carries a non-degenerate four-form $\Omega$ which may locally be expressed as

$$
\Omega=\omega_{I} \wedge \omega_{I}+\omega_{J} \wedge \omega_{J}+\omega_{K} \wedge \omega_{K} .
$$


In dimension eight and above one says that $Q$ is quaternionic Kähler if the fundamental form is parallel, $\nabla^{\mathrm{LC}} \Omega=0$. This implies that $\Omega$ is closed. In dimensions 12 and higher $d \Omega=0$ is actually equivalent to the quaternionic Kähler condition [36]. Gray [21] showed that the stabiliser of $\Omega$ under the action of $G L(4 n, \mathbb{R}), n>1$, is the compact group $S p(n) S p(1)$ and so $\Omega$ determines the metric $g$. In dimension four, these considerations no longer hold and a quaternionic Kähler manifold is instead defined to be an oriented Riemannian manifold which is Einstein and self-dual.

If the subbundle $\mathcal{G}$ can be globally trivialised by $I, J, K$ and these almost complex structures are integrable then we have a hyper-Hermitian manifold. This will then be hyperKähler provided that the two-forms $\omega_{I}$, etc., are closed.

In [37] it was shown that to any quaternionic Kähler manifold $Q^{4 n}$ of positive scalar curvature one may associate a hyperKähler manifold $M^{4 n+4}=\mathcal{U}(Q)$ which acts as a hyperKähler generalisation of the twistor space; this is known as the Swann bundle and may be written as $\mathcal{U}(Q)=$ $\mathbb{R}_{>0} \times \mathcal{S}$, where $\mathcal{S}$ is the bundle of triples $(I, J, K)$. Conversely given a $(4 n+4)$-dimensional hyperKähler manifold $M$ admitting a special type of $S U(2)$-action then a version of the Marsden-Weinstein reduction produces a quaternionic-Kähler manifold of positive scalar curvature; this latter reduction process can be realised in terms of multi-moment maps.

The relevant type of $S U(2)$-symmetry often arises due to the presence of a vector field $X$ on $\left(M^{4 n+4}, g, I, J, K\right)$, a special homothety (cf. [33]), with the following properties:

$$
\mathcal{L}_{X} g=g, \quad \mathcal{L}_{I X} g=0, \quad \mathcal{L}_{I X} I=0, \quad \mathcal{L}_{I X} J=-K, \quad \mathcal{L}_{I X} K=J, \quad \text { etc. }
$$

Special homotheties generate a local action of $\mathbb{H}^{*}$ and in good cases the vector fields $I X, J X, K X$ integrate to give an action of $S U(2)$ which is necessarily locally free.

Proposition 4.2 (Madsen [28]). Let $\left(M^{4 n+4}, g, I, J, K\right)$ be a hyperKähler manifold, and $X$ a special homothety. If IX, JX, KX generate a locally free action of $S U(2)$ then this action preserves $\Omega$ and a multi-moment map $v: M \rightarrow \mathbb{R} \cong$ $\mathcal{P}_{\mathfrak{s u}(2), 2}^{*}$ is given by

$$
v=-3\|X\|^{4}
$$

Any non-zero $t \in v(M)$ is a regular value. The level sets correspond to $\|X\|$ is constant and the results of [37] show that $M / / v, t S U(2)$ is a quaternionic Kähler orbifold of positive scalar curvature.

\subsection{Holonomy Spin(7)}

A $\operatorname{Spin}(7)$ structure is a geometry modelled on the form $\Phi_{0}$ of equation (2.3). 


\section{Closed Forms AND MULTI-MOMENT MAPS}

Definition 4.3. An eight-manifold $M$ has a $\operatorname{Spin}(7)$-structure if there is a form $\Phi \in \Omega^{4}(M)$ such that $\left(T_{x} M, \Phi_{x}\right)$ is linearly isomorphic to $\left(\mathbb{R}^{8}, \Phi_{0}\right)$ for each $x \in M$.

Now $\Phi$ determines a volume form and Riemannian metric on $M$ via the relations

$$
\left.\Phi^{2}=14 \text { vol }, \quad((X \wedge Y)\lrcorner \Phi\right)^{2} \wedge \Phi=6\|X \wedge Y\|_{g}^{2} \text { vol } .
$$

Comparing with $\Phi_{0}$ we see that vol and $g$ correspond to the standard volume $\operatorname{vol}_{0}=e_{12345678}$ and metric $g_{0}=\sum_{i=1}^{8} e_{i}^{2}$ on $\mathbb{R}^{8}$. We also see that $\Phi$ is a self-dual four-form $* \Phi=\Phi$. In particular, a closed $\operatorname{Spin}(7)$-structure has $\Phi$ harmonic and it follows that the holonomy group of $g$ is contained in $\operatorname{Spin}(7)$. This is one of the two exceptional holonomies in the Berger classification $[5,6]$. Examples of metrics with holonomy exactly $\operatorname{Spin}(7)$ are not easy to find. Local existence was proved by Bryant [8], the first complete examples were produced by Bryant and Salamon [9] and the first compact examples were found by Joyce [25]. The complete examples produced by Bryant \& Salamon have many symmetries, in fact the symmetry group acts with cohomogeneity one, so the principal orbit is of codimension one. Further systematic study of cohomogeneity one examples with compact symmetry group has been made by Reidegeld [34, 35]. One sees that many of the examples and candidates have compact symmetry groups of rank 3, so an interesting class of $\operatorname{Spin}(7)$-manifolds are those with $T^{3}$-symmetry.

Given a closed $\operatorname{Spin}(7)$-structure $(M, \Phi)$ with free $T^{3}$-symmetry, fix a basis $U_{1}, U_{2}, U_{3}$ for $\mathfrak{t} \cong \mathbb{R}^{3}$. Then we have the following two-forms on $M$ :

$$
\left.\left.\left.\left.\left.\left.\omega_{1}=U_{2}\right\lrcorner U_{3}\right\lrcorner \Phi, \quad \omega_{2}=U_{3}\right\lrcorner U_{1}\right\lrcorner \Phi, \quad \omega_{3}=U_{1}\right\lrcorner U_{2}\right\lrcorner \Phi .
$$

These forms are all closed, by the Lemma 3.4.

To see the structure of these forms, we consider the geometry of $\left(\mathbb{R}^{7}, \Phi_{0}\right)$. Isolating $e_{1}$ in the expression (2.3) for $\Phi_{0}$ we have

$$
\begin{aligned}
\Phi_{0}=e_{1} & \wedge\left(e_{234}+e_{256}+e_{278}+e_{357}-e_{368}-e_{458}-e_{467}\right) \\
& +e_{5678}+e_{3478}+e_{3456}+e_{2468}-e_{2457}-e_{2367}-e_{2358} \\
=e_{1} & \wedge \varphi_{0}+*_{7} \varphi_{0},
\end{aligned}
$$

where we recognise $\varphi_{0}$ on $V_{7}=\left\langle E_{2}, \ldots, E_{8}\right\rangle$ as a rewritten version of $\phi_{0}$ in (2.2) and $*_{7}$ is the Hodge star operator on $V_{7}$ with respect to the induced metric and volume. In particular, we see that the stabiliser of $e_{1}$ under the action of $\operatorname{Spin}(7)$ is the stabiliser of $\varphi_{0}$ which is the exceptional group $G_{2}$. The orbit of $e_{1}$ under the action of the compact group $\operatorname{Spin}(7)$ is thus of dimension $\operatorname{dim} \operatorname{Spin}(7)-\operatorname{dim} G_{2}=21-14=7$. As the Spin(7)-action preserves the metric $g_{0}$, we conclude that $\operatorname{Spin}(7)$ acts transitively on the 
unit sphere $S^{7} \subset \mathbb{R}^{8}$. Thus for any unit vector $v \in \mathbb{R}^{8}$, we have that $\left.v\right\lrcorner \Phi_{0}$ is a $G_{2}$-form on $v^{\perp}$. to get

We may now repeat this argument, isolating $e_{2}$ in the expression for $\varphi_{0}$

$$
\begin{aligned}
\varphi_{0}=e_{2} & \wedge\left(e_{34}+e_{56}+e_{78}\right) \\
& +e_{357}-e_{368}-e_{458}-e_{468} \\
= & e_{2} \wedge \omega+\psi_{+} .
\end{aligned}
$$

This time $\omega$ is a symplectic form on $V_{6}=\left\langle E_{3}, \ldots, E_{8}\right\rangle$ and $\psi_{+}$is the real part of the complex volume form $\left(e_{3}+i e_{4}\right) \wedge\left(e_{5}+i e_{6}\right) \wedge\left(e_{7}+i e_{8}\right)$ on $V_{6} \cong$ $\mathbb{R}^{6} \cong \mathbb{C}^{3}$. The stabiliser of $e_{2}$ under the action of $G_{2}$ is the stabiliser of the pair $\left(\omega, \psi_{+}\right)$which is $S U(3)$. The orbit of $e_{2} \in V_{7}$ has dimension $\operatorname{dim} G_{2}-$ $\operatorname{dim} S U(3)=14-8=6$ and is just the unit sphere $S^{6} \subset V_{7}$. Finally, the orbit of $e_{3} \in V_{6}$ under the action of $S U(3)$ is $S^{5}=S U(3) / S U(2)$. This demonstrates the following well-known result, cf. Bryant [8].

Lemma 4.4. Spin (7) acts transitively on orthonormal pairs $\left(v_{1}, v_{2}\right)$ and orthonormal triples $\left(v_{1}, v_{2}, v_{3}\right)$ in $\mathbb{R}^{8}$.

In particular, $\operatorname{Spin}(7)$ acts transitively on the sets of unit length simple bivectors $u_{1} \wedge u_{2}$ and unit length simple trivectors $u_{1} \wedge u_{2} \wedge u_{3}$ on $\mathbb{R}^{8}$. Using the first of these statements, we describe the form $\omega_{3}$ of (4.3) as pointwise corresponding to a multiple of $\left.\left(E_{1} \wedge E_{2}\right)\right\lrcorner \Phi_{0}=e_{34}+e_{56}+e_{78}$. Thus each $\omega_{i}$ is a two-form of rank 6 .

Furthermore, suppose that $v: M \rightarrow \mathbb{R}$ is a multi-moment map for the action of $T^{3}$. Then $\left.d v=\left(U_{1} \wedge U_{2} \wedge U_{3}\right)\right\lrcorner \Phi$ which corresponds to a multiple of $\left.\left(E_{1} \wedge E_{2} \wedge E_{3}\right)\right\lrcorner \Phi_{0}=e_{4}$. This implies that on a level set $v^{-1}(t) \subset M$, the pull-back $i^{*} \omega_{3}$ of $\omega_{3}$ under the inclusion map $i$ corresponds to a multiple of $e_{56}+e_{78}$. One may prove that the $T^{3}$-invariant forms $i^{*} \omega_{1}, i^{*} \omega_{2}, i^{*} \omega_{3}$ each vanishes on $U_{1}, U_{2}$ and $U_{3}$ and thus they descend to two-forms on the four-manifold $N_{t}=M / / v, t T^{3}$. The following terminology will be used:

Definition 4.5. A triple of $\sigma_{1}, \sigma_{2}, \sigma_{3}$ of symplectic structures on a manifold $N$ of dimension four is weakly coherent if the forms are pointwise linearly independent, define the same orientation and the pairing $\sigma_{i} \wedge \sigma_{j}$ has definite sign.

A more detailed analysis of our situation gives the following description of the quotients $N_{t}$.

Proposition 4.6 (Madsen [27]). Let $(M, \Phi)$ be a closed Spin(7)-structure. Suppose $T^{3}$ acts freely on $M$ preserving $\Phi$ and with a multi-moment map $v$. Then for each $t \in v(M)$, the four-manifold $N_{t}=M / / v, t T^{3}$ admits a real-analytic weakly coherent triple of symplectic structures $\sigma_{1}, \sigma_{2}, \sigma_{3}$. 


\section{Closed Forms AND MULTI-MOMENT MAPS}

The fact that the quotient geometry is real-analytic follows from the remark that $T^{3}$ acts by isometries and that closed $\operatorname{Spin}(7)$ structures are Ricci-flat. It follows that the vector fields $U_{i}$ are real-analytic.

To fully describe the relationship between the geometries of $M$ and $N_{t}$, one may construct a connection one-form $\theta \in \Omega^{1}(M, \mathfrak{t})$ as follows. Let $G=$ $\left(g_{i j}\right)$ with $g_{i j}=g\left(U_{i}, U_{j}\right)$ and let $U^{b}=\left(U_{1}^{b}, U_{2}^{b}, U_{3}^{b}\right)$, where $U_{i}^{b}=g\left(U_{i}, \cdot\right)$. Then $\theta$ is given by

$$
\theta=U^{b} G^{-1}
$$

This satisfies $\theta_{i}\left(U_{j}\right)=\delta_{i j}$ as required. The matrix $G$ here turns out to be determined the geometry on $N_{t}$.

Lemma 4.7 (Madsen [27]). $G^{-1}=h^{2} Q$, where $h=1 / \sqrt{\operatorname{det} G}$ and $Q=\left(q_{i j}\right)$ is the matrix given by

$$
\sigma_{i} \wedge \sigma_{j}=2 q_{i j} \mathrm{vol}
$$

on $N_{t}$.

Here vol denotes the volume form on $N_{t}$ induced from the quotient.

As $v^{-1}(t)$ is a $T^{3}$-bundle over $N_{t}$ its curvature $F=d \theta$ is a closed form with integral periods. We write $F \in \Omega_{\mathbb{Z}}^{2}(N, \mathfrak{t})$ to indicate this. Conversely, given such an $F$, one may construct a principal $T^{3}$-bundle over $N$ with curvature $F$. Madsen shows that $F$ satisfies the following symmetry condition

$$
F_{i} \wedge \sigma_{j}=F_{j} \wedge \sigma_{i}, \quad \text { for all } i, j .
$$

It turns out that this data is sufficient to invert the construction.

Theorem 4.8 (Madsen [27]). Suppose $N$ is a connected four-manifold with a real-analytic weakly coherent symplectic triple $\left(\sigma_{1}, \sigma_{2}, \sigma_{3}\right)$ and volume form vol, with the same orientation as $\sigma_{i}^{2}$. For each real-analytic $F \in \Omega_{\mathbb{Z}}^{2}(N, \mathfrak{t})$ satisfying (4.5) there is a unique maximal connected closed $\operatorname{Spin}(7)$-structure $(M, \Phi)$ with $T^{3}$-symmetry and multi-moment map $v$, such that $\left(N, \sigma_{i}, \mathrm{vol}, F\right)$ is the reduction of $M$ at level 0 .

The idea of the proof is to construct a $G_{2}$-geometry on the $T^{3}$-bundle $P \rightarrow N$ determined by $F$. One then uses a modified variant of the Hitchin flow to extend this to a closed $\operatorname{Spin}(7)$-geometry on a maximal open subset of $P \times \mathbb{R}$. Explicit examples of this construction are given in [27]. Note that even the reduction of $\mathbb{R}^{8}$ by the maximal torus of $\operatorname{Spin}(7)$ gives non-trivial tri-symplectic geometries on $\mathbb{R}^{4}$ that are not hyperKähler.

\section{6 $\mathrm{G}_{2}$-manifolds}

A $G_{2}$-structure on a seven-manifold $M$ is a choice of three-form $\phi \in \Omega^{3}(M)$ which on each tangent space $T_{x} M$ is linearly equivalent to the form $\phi_{0}$ 
of (2.2) on $\mathbb{R}^{7}$. As in the $\operatorname{Spin}(7)$ case, the form $\phi$ determines a volume form and metric on $M$ via the relation

$$
(X\lrcorner \phi) \wedge(Y\lrcorner \phi) \wedge \phi=6 g(X, Y) \text { vol . }
$$

To interpret this formula, note that $g$ is required to be positive definite, which determines the sign of vol and the conformal class of $g$. Now the fact that vol is required to be of unit length with respect to $g$, fixes the conformal factor: scaling $g$ by $f^{2}>0$ scales vol by $f^{7}$ and the right-hand side of (4.6) scales by $f^{9}$ (cf. [8, 26]).

The form $\phi_{0}$ is stable in the sense of Definition 2.3 and so is its Hodge dual, the four-form $*_{7} \phi_{0}$. However, the stabiliser of $*_{7} \phi_{0}$ is $G_{2} \times \mathbb{Z}_{2}$ rather than just $G_{2}$, as may be seen by noting that $-\phi_{0}$ defines the opposite orientation on $\mathbb{R}^{7}$.

There are a number of classes of $G_{2}$-structures with closed four-form that have particular interest for us.

Definition 4.9. Let $(M, \phi)$ be a $G_{2}$-structure. The structure is

(i) cosymplectic if $d *_{7} \phi=0$,

(ii) parallel if $d \phi=0$ and $d *_{7} \phi=0$,

(iii) nearly parallel if $d \phi=4 *_{7} \phi$.

Any oriented hypersurface $M$ of a closed $\operatorname{Spin}(7)$-manifold $Y$ carries a cosymplectic $G_{2}$-structure. Indeed write $i: M \rightarrow Y$ for the inclusion and let $\mathrm{N}$ be a unit normal to the hypersurface, then formula (4.4) gives

$$
\left.\Phi\right|_{Y}=\mathbf{N}^{b} \wedge \phi+*_{7} \phi
$$

with $\left.\phi=i^{*}(\mathbf{N}\lrcorner \Phi\right)$ defining the $G_{2}$-structure. This gives $d *_{7} \phi=d i^{*} \Phi=$ $i^{*} d \Phi=0$, showing that the $G_{2}$-structure is cosymplectic.

Parallel $G_{2}$-structures are so-called because the equations $d \phi=0$ and $d *_{7} \phi=0$ imply that $\phi$ is parallel for the Levi-Civita connection of $g$, as shown by Fernández and Gray [15]. This implies that $g$ is Ricci-flat, cf. Bonan [7], and that the holonomy is contained in $G_{2}$.

Given a parallel $G_{2}$-structure $(M, \phi)$ we may consider $Y=S^{1} \times M$ with the four-form $\Phi=\theta \wedge \phi+*_{7} \phi$, and see that $\Phi$ gives a closed $\operatorname{Spin}(7)$ structure. If $T^{2}$ acts on $M$ preserving $\phi$, then it also preserves $*_{7} \phi$. Now much as in Section 4.2, a multi-moment map $v$ for $T^{2}$ on $(M, \phi)$ gives a multi-moment map $\tilde{v}$ for $T^{3}=S^{1} \times T^{2}$ acting on $\left(Y=S^{1} \times M, \Phi\right)$.

The theory of reductions of $T^{3}$-invariant closed $\operatorname{Spin}(7)$-structures, discussed in the previous section, may now be applied to $T^{2}$-invariant parallel $G_{2}$-structures. This gives that $N_{t}=M / / v, t T^{2}$ carries coherent triple of symplectic structures $\sigma_{0}, \sigma_{1}, \sigma_{2}$, meaning that they are weakly coherent and $\sigma_{0} \wedge \sigma_{i}=0$ for $i=1,2$. Also the curvature associated to the $S^{1}$-factor of $Y$ is trivial, so $v^{-1}(t) \rightarrow N_{t}$ is a $T^{2}$-bundle with curvature form $F \in \Omega_{\mathbb{Z}}^{2}\left(N_{t}, \mathfrak{t}\right)$, 


\section{Closed Forms AND MULTI-MOMENT MAPS}

whose self-dual part has no $\sigma_{0}$-component, and which satisfies the condition (4.5). A direct description of this situation is given in [30].

The third class of Definition 4.9 is the manifolds of nearly parallel $G_{2}$ structures. These also go under the name of weak holonomy $G_{2}$ in the terminology of Gray [22], who showed that the associated metric $g$ is Einstein with positive scalar curvature. The simplest example of such a $G_{2}$ structure is the unit sphere $S^{7} \subset \mathbb{R}^{8}$, with the geometry induced from the flat $\operatorname{Spin}(7)$-structure. The discussion above shows that $S^{7}=\operatorname{Spin}(7) / G_{2}$ so the maximal torus $T^{3}$ acts preserving this geometry. The $\operatorname{Spin}(7)$-geometry on $\mathbb{R}^{8}$ can be recovered as a warped product.

Indeed, suppose $(M, \phi)$ is a nearly parallel $G_{2}$-structure. Put $C(M)=$ $\mathbb{R}_{>0} \times M$ with the form

$$
\Phi_{C}=s^{3} d s \wedge \phi+s^{4} *_{7} \phi,
$$

where $s$ is the parameter on $\mathbb{R}_{>0}$. At level $s$, the induced structure on $\{s\} \times M$ is given by $s^{3} \phi$, which is a $G_{2}$-structure with metric $s^{2} g$. This shows that at each point $\Phi_{C}$ is linearly isomorphic to the $\operatorname{Spin}(7)$-form $\Phi_{0}$ of (2.3); so $\Phi_{C}$ defines a $\operatorname{Spin}(7)$-structure on $C(M)$. As $d \phi=4 *_{7} \phi$, the four-form $*_{7} \phi$ is closed and we find that

$$
d \Phi_{C}=-s^{3} d s \wedge d \phi+4 s^{3} d s \wedge *_{7} \phi+s^{4} d *_{7} \phi=0 .
$$

Thus $\left(C(M), \Phi_{C}\right)$ is a closed $\operatorname{Spin}(7)$-structure. Its metric is the warped product $g_{C}=d s^{2}+s^{2} g$. This construction was used by Bär [3] to relate the Killing spinors of $(M, g)$ to parallel spinors of $\left(C(M), g_{C}\right)$. For more on the Killing spinor approach to these $G_{2}$-structures see [4, 18].

Now any symmetry of $(M, \phi)$ induces a symmetry of $\left(C(M), \Phi_{C}\right)$ that preserves $s$. Thus a nearly parallel $G_{2}$-structure with $T^{3}$-symmetry corresponds to a certain class of $T^{3}$-invariant closed $\operatorname{Spin}(7)$-structures. As in the previous section, let $U_{1}, U_{2}, U_{3}$ be vector fields generating the $T^{3}$-action. For $u=U_{1} \wedge U_{2} \wedge U_{3}$, the nearly parallel condition and the extended Cartan formula (Lemma 3.4) give

$$
\left.u\lrcorner *_{7} \phi=\frac{1}{4} u\right\lrcorner d \phi=-\frac{1}{4} d(\phi(u)) .
$$

Thus $v=-\frac{1}{4} \phi(u)$ is a multi-moment map for the $T^{3}$-action on $(M, * \phi)$. Also we have that

$$
\begin{aligned}
u\lrcorner \Phi_{C} & \left.=-s^{3} d s \phi(u)+s^{4} u\right\lrcorner *_{7} \phi \\
& =-\frac{1}{4}\left(d\left(s^{4} \phi(u)\right)\right) .
\end{aligned}
$$

So $v_{C}=s^{4} v$ is a multi-moment map for the action on the cone $\left(C(M), \Phi_{C}\right)$.

Now for general $t$, the level set $v_{C}^{-1}(t)$ consists of the $(s, m)$ such that $s^{4} v(m)=t$. Since $s \in \mathbb{R}_{>0}$, this relation simplifies when $t=0$ and we have 
that

$$
v_{C}^{-1}(0)=C\left(v^{-1}(0)\right)
$$

We will therefore only consider the reductions at level 0 .

The reduction $N_{C}=v_{C}^{-1}(0) / T^{3}$ of the cone carries a weakly coherent triple of symplectic forms $\sigma_{1}, \sigma_{2}, \sigma_{3}$ with for example $\left.\left.\pi_{C}^{*} \sigma_{3}=i_{C}^{*}\left(U_{1}\right\lrcorner U_{2}\right\lrcorner \Phi_{C}\right)$, where $i_{C}: C(M)_{0}=v_{C}^{-1}(0) \hookrightarrow C(M)$ is the inclusion and $\pi_{C}: C(M)_{0} \rightarrow N_{C}$ is the projection. However,

$$
\begin{aligned}
\pi_{C}^{*} \sigma_{3} & \left.\left.=i_{C}^{*}\left(U_{1}\right\lrcorner U_{2}\right\lrcorner \Phi_{C}\right) \\
& \left.\left.\left.\left.=i_{C}^{*}\left(s^{3} d s \wedge\left(U_{1}\right\lrcorner U_{2}\right\lrcorner \phi\right)+\frac{1}{4} U_{1}\right\lrcorner U_{2}\right\lrcorner d \phi\right) \\
& =i_{C}^{*} d\left(\frac{1}{4} s^{4} \pi^{*} \eta_{3}\right),
\end{aligned}
$$

where $\left.\left.\pi^{*} \eta_{3}=i^{*}\left(U_{1}\right\lrcorner U_{2}\right\lrcorner \phi\right)$, with $i: M_{0}=v^{-1}(0) \hookrightarrow M$ the inclusion and $\pi: M_{0} \rightarrow N=M / /{ }_{\nu} T^{3}$ the projection. The fact that $\sigma_{3}$ is non-degenerate on $N_{C}=C(N)$ then corresponds to $\eta_{3}$ being a contact structure on $N$.

In this way, we see that the reduction $N=M / / v T^{3}$ carries a pointwise linearly independent triple $\left(\eta_{1}, \eta_{2}, \eta_{3}\right)$ of contact structures. The condition that the $\sigma_{i}$ are weakly coherent corresponds to the requirements that the forms $\eta_{i} \wedge d \eta_{i}$ define the same orientation and that the symmetric matrix with entries corresponding to $\eta_{i} \wedge d \eta_{j}+\eta_{j} \wedge d \eta_{i}$ is positive definite. Thus one example is provided by taking $N=S^{3}=S U(2)$, with $d \eta_{1}=-2 \eta_{2} \wedge \eta_{3}$ etc. Conversely the standard basis of one forms for $S L(2, \mathbb{R})$ does not give a weakly coherent triple.

The remaining data for the $\operatorname{Spin}(7)$-geometry are the curvature forms $F_{1}, F_{2}, F_{3}$ satisfying (4.5). These forms are invariant under the action of the Euler vector field on $N_{C}$, so they have the form $F=d \log s \wedge a_{i}+b_{i}$, with $a_{i} \in \Omega^{1}(N)$ closed and $b_{i} \in \Omega_{\mathbb{Z}}^{2}(N)$. Equation (4.5) becomes

$$
a_{i} \wedge d \eta_{j}+4 b_{i} \wedge \eta_{j}=a_{j} \wedge d \eta_{i}+4 b_{j} \wedge \eta_{i}, \quad \text { for all } i, j \text {. }
$$

Thus $N$ carries three-contact forms and the closed forms $a_{1}, \ldots, b_{3}$.

\subsection{PSU(3)-structures}

A $\operatorname{PSU}(3)$-structure on an oriented eight-manifold $M$ is a three-form $\rho \in$ $\Omega^{3}(M)$ pointwise modelled on $\rho_{0}$ of equation (2.4). These geometries were studied by Witt [39, 40]. Such a form $\rho$ determines a metric $g$ and the fiveform $* \rho$. A PSU(3)-structure is said to be harmonic if $d \rho=0$ and $d * \rho=0$.

The case when a harmonic PSU(3)-structure admits a free two-torus symmetry with multi-moment map $v$ was discussed in [28] reinterpreting some results of Witt. In this case one has four two-forms given by

$$
\begin{gathered}
\left.\left.\left.\left.\omega_{0}=-(d v)^{\sharp}\right\lrcorner U_{1}\right\lrcorner U_{2}\right\lrcorner * \rho, \quad \omega_{1}=U_{1}\right\lrcorner \rho, \\
\left.\left.\left.\left.\omega_{2}=U_{2}\right\lrcorner \rho, \quad \omega_{3}=U_{1}\right\lrcorner U_{2}\right\lrcorner \alpha^{\sharp}\right\lrcorner * \rho,
\end{gathered}
$$




\section{Closed Forms AND MULTI-MOMENT MAPS}

where $\alpha$ is contraction of $\rho$ by $\omega_{0}$. At a regular value $t$ of $v$, these forms induce two-forms $\sigma_{i}$ on the reduction $N=M / / v, t T^{2}$ and $\alpha$ induces a oneform $a$. Together $\left(a, \sigma_{1}, \sigma_{2}, \sigma_{3}\right)$ give $N$ the structure of an $S U(2)$-manifold, see Conti and Salamon [13], and a conformal scaling of $a \wedge \sigma_{0}$ can be 2plectic.

\subsection{Homogeneous k-plectic manifolds}

Suppose $(M, \alpha)$ is a closed geometry of degree $r=k+1$ with a group $G$ of symmetries that acts transitively on $M$. Then the equivariant map $\Psi: M \rightarrow$ $Z^{r}(\mathfrak{g})$ given by (3.10) has image a single $G$-orbit in $Z^{r}(\mathfrak{g})$. Conversely, we may use equation (3.10) to define closed geometries that map to a given orbit $G \cdot \Psi \subset Z^{r}(\mathfrak{g})$, as follows. Let $K_{\Psi}$ be the connected subgroup of $G$ with Lie algebra $\operatorname{ker} \Psi=\{X \in \mathfrak{g}: X\lrcorner \Psi=0\}$. Then for each closed subgroup $H$ of $G$ containing $K_{\Psi}$, equation (3.10) gives a well-defined closed $r$-form $\alpha$ on $M=G / H$.

Now suppose that $\Psi=d_{\mathcal{P}} \beta$ for some $\beta \in \mathcal{P}_{\mathfrak{g}}^{*}$. If the map $d_{\mathcal{P}}$ is injective, then the orbits $G \cdot \Psi$ and $G \cdot \beta$ are identified and the map $\Psi: M \rightarrow Z^{r}(\mathfrak{g})$ may now be interpreted as a map $v: M \rightarrow \mathcal{P}_{\mathfrak{g}}^{*}$. Injectivity of $d_{\mathcal{P}}$ is equivalent to the condition $b_{r-1}(\mathfrak{g})=0$ and the proof of Theorem 3.14 shows that $v$ is a multi-moment map for the action of $G$.

Theorem 4.10. Suppose $G$ is a connected Lie group with $b_{k}(\mathfrak{g})=0$. Let $\mathcal{O}=$ $G \cdot \beta \subset \mathcal{P}_{\mathfrak{g}}^{*}$ be an orbit of $G$ acting on the dual of the kth Lie kernel. Then there are homogeneous closed geometries $(G / H, \alpha)$, with $\alpha \in \Omega^{k+1}(G / H)$ corresponding to $\Psi=d_{\mathcal{P}} \beta$, such that $\mathcal{O}$ is the image of $G / H$ under the (unique) multi-moment map $v$.

The closed geometry may be realised on the orbit $\mathcal{O}$ itself if and only if

$$
\operatorname{stab}_{\mathfrak{g}} \beta=\operatorname{ker}\left(d_{\mathcal{P}} \beta\right) .
$$

In this situation, the orbit is $k$-plectic and $v$ is simply the inclusion $\mathcal{O} \hookrightarrow \mathcal{P}_{\mathfrak{g}}^{*}$.

Proof. It only remains to prove the assertions of the last paragraph of the theorem. We have $\mathcal{O}=G / K$ with $K=\operatorname{stab}_{G} \beta$, a closed subgroup of $G$. Now equation (4.7), shows that $K$ has Lie algebra $\operatorname{ker}\left(d_{\mathcal{P}} \beta\right)$, so the component of the identity $K^{0}$ of $K$ is $K^{0}=K_{\Psi}$ for $\Psi=d_{\mathcal{P}} \beta$. In particular, $\Psi$ vanishes on elements of $\mathfrak{k}$ and induces a well-defined form on $T_{\beta} \mathcal{O}=\mathfrak{g} / \mathfrak{k}$. The result now follows.

Remark 4.11. In the case when $r=2$, condition (4.7) is automatic and we get the result of Kirillov-Kostant-Souriau that each orbit of $\mathfrak{g}^{*}$ is symplectic.

Example 4.12. Suppose $G$ is a $(k, k+1)$-trivial Lie group. Then, taking $H=$ $\{e\}$, we see that every $\Psi \in Z^{k+1}(\mathfrak{g})$ gives rise to a closed geometry on $G$ with multi-moment map whose image is diffeomorphic to the G-orbit of $\Psi$. 


\section{Cohomology of Lie algebras}

Recall from Section 3.2 that for a closed geometry of degree $r$, multi-moment maps exist and are unique for any symmetry group $G$ which is $(r-1, r)$ trivial. This condition means that the cohomology groups $H^{k}(\mathfrak{g})$ of $\mathfrak{g}$ are trivial in degrees $k=r-1$ and $r$. While this is a concise statement, it is not clear which, if any algebras, satisfy these conditions. In this section, we will discuss some techniques to gain more information and show that there are in fact many such algebras.

Note first that the definitions (3.8) and (3.9) give $H^{1}(\mathfrak{g})=\operatorname{ker} d \leqslant \mathfrak{g}^{*}$. As $d$ is dual to the Lie bracket $L=[\cdot, \cdot]: \Lambda^{2} \mathfrak{g} \rightarrow \mathfrak{g}$, the vanishing of $b_{1}(\mathfrak{g})=$ $\operatorname{dim} H^{1}(\mathfrak{g})$ is equivalent to the surjectivity of $L$. This says that $b_{1}(\mathfrak{g})=0$ if and only if $\mathfrak{g}$ is equal to its derived algebra $\mathfrak{g}^{\prime}=[\mathfrak{g}, \mathfrak{g}]$. Indeed $b_{1}(\mathfrak{g})$ is exactly the codimension of $\mathfrak{g}^{\prime}$ in $\mathfrak{g}$. Lie algebras with $\mathfrak{g}=\mathfrak{g}^{\prime}$ are called perfect. Any semi-simple Lie algebra is perfect, but other examples may be constructed as the semi-direct product $\mathfrak{h} \ltimes V$ of a semi-simple algebra $\mathfrak{h}$ with a faithful representation $V$. For example, the group of isometries of $\mathbb{R}^{n}$ with the standard flat metric is perfect for each $n \geqslant 3$.

Interpretation of the vanishing of higher Betti numbers is more complicated. We gave some of the vanishing properties satisfied by compact simple Lie groups in Section 3.2. In particular, these are $(1,2)$-trivial, leading to the usual existence and uniqueness results for symplectic moment maps. Furthermore any $(1,2)$-trivial algebra is semi-simple. The structure of the (2,3)-trivial groups was described in [30] and classification results in small dimensions given in the same paper and in [29]. In particular, we found that $(2,3)$-trivial Lie algebras are always solvable, meaning that $\mathfrak{g}^{m}=\{0\}$ for some $m>0$, where $\mathfrak{g}^{m}=\left[\mathfrak{g}^{m-1}, \mathfrak{g}^{m-1}\right]$ is the $m$ th derived algebra of $\mathfrak{g}$.

The general structure theory of Lie algebras says that any $\mathfrak{g}$ has a maximal solvable ideal $\mathfrak{r}$, the solvable radical, and that the quotient $\mathfrak{g} / \mathfrak{r}$ is semisimple. In addition, for any solvable Lie algebra $\mathfrak{h}$ the derived algebra $\mathfrak{k}=\mathfrak{h}^{\prime}$ is always nilpotent, meaning that $\mathfrak{k}_{m}=\{0\}$ for some $m>0$, where $\mathfrak{k}_{m}=\left[\mathfrak{k}, \mathfrak{k}_{m-1}\right]$ and $\mathfrak{k}_{1}=\mathfrak{k}^{\prime}$. In [30], a result of Hochschild and Serre [24] relating the cohomologies of $\mathfrak{g}, \mathfrak{r}$ and $\mathfrak{g} / \mathfrak{r}$ was used in [30] to prove:

Lemma 5.1. Any non-zero Lie algebra with $b_{3}(\mathfrak{g})=0$ is solvable and thus has $b_{1}(\mathfrak{g}) \neq 0$. Such $a \mathfrak{g}$ is not nilpotent unless $\mathfrak{g}=\mathbb{R}$ or $\mathfrak{g}=\mathbb{R}^{2}$.

The essential point is that the semi-simple algebra $\mathfrak{g} / \mathfrak{r}$ has $b_{3}$ non-zero, and this feeds through to $b_{3}(\mathfrak{g})$ if $\mathfrak{r} \neq \mathfrak{g}$. For a solvable algebra $\mathfrak{g}^{\prime}$ is strictly smaller than $\mathfrak{g}$, so $b_{1}(\mathfrak{g}) \neq 0$.

The $(2,3)$-trivial algebras were then found to be exactly those solvable $\mathfrak{g}$ such that the derived algebra $\mathfrak{k}=\mathfrak{g}^{\prime}$ has codimension 1 in $\mathfrak{g}$ and such that $\mathfrak{g} / \mathfrak{k}$ acts invertibly on the cohomology groups $H^{i}(\mathfrak{k})$, for $i=1,2,3$. 


\section{Closed Forms AND MULTI-MOMENT MAPS}

\section{1 (3,4)-trivial Lie algebras}

Let us work towards a description of $(3,4)$-trivial Lie algebras. As a first result we consider direct sums of algebras.

Proposition 5.2. A non-trivial direct sum $\mathfrak{g}=\mathfrak{h}_{1}+\mathfrak{h}_{2}$ has $b_{3}(\mathfrak{g})=0$ if and only if each summand $\mathfrak{h}_{i}$ is $(2,3)$-trivial. Consequently, $\mathfrak{g}$ is product of at most two summands.

The direct sum $\mathfrak{g}=\mathfrak{h}_{1}+\mathfrak{h}_{2}$ is $(3,4)$-trivial if and only if each summand is $(2,3,4)$-trivial.

Proof. This is almost direct from the Künneth formula, which gives the following sum of positive terms:

$$
b_{3}(\mathfrak{g})=b_{3}\left(\mathfrak{h}_{1}\right)+b_{3}\left(\mathfrak{h}_{2}\right)+b_{2}\left(\mathfrak{h}_{1}\right) b_{1}\left(\mathfrak{h}_{2}\right)+b_{1}\left(\mathfrak{h}_{1}\right) b_{2}\left(\mathfrak{h}_{2}\right) .
$$

Thus $b_{3}(\mathfrak{g})=0$ immediately gives $b_{3}\left(\mathfrak{h}_{i}\right)=0$. However, by Lemma 5.1 we known that $b_{1}\left(\mathfrak{h}_{i}\right) \neq 0$, so the vanishing of $b_{3}(\mathfrak{g})$ also gives $b_{2}\left(\mathfrak{h}_{i}\right)=0$.

Now a similar argument, given in [30], shows that (2,3)-trivial algebras are not direct sums of smaller ideals. Thus the summands $\mathfrak{h}_{i}$ are not direct sums and $\mathfrak{g}$ has at most two summands.

In the second case, the Künneth formula gives

$$
b_{4}(\mathfrak{g})=b_{4}\left(\mathfrak{h}_{1}\right)+b_{4}\left(\mathfrak{h}_{2}\right)+b_{2}\left(\mathfrak{h}_{1}\right) b_{2}\left(\mathfrak{h}_{2}\right)+b_{3}\left(\mathfrak{h}_{1}\right) b_{1}\left(\mathfrak{h}_{2}\right)+b_{1}\left(\mathfrak{h}_{1}\right) b_{3}\left(\mathfrak{h}_{2}\right),
$$

so the extra condition $b_{4}(\mathfrak{g})=0$ forces $b_{4}\left(\mathfrak{h}_{i}\right)=0$ too.

The converse statements are immediate from (5.1) and (5.2).

To go further and study other cases we need to use stronger techniques. Our main tool will be the Hochschild-Serre spectral sequence of a Lie algebra $\mathfrak{g}$ with respect to an ideal $\mathfrak{k}$. We will consider the case when the $\mathfrak{k}$ contains the derived algebra $\mathfrak{g}^{\prime}$. Then the quotient algebra $\mathfrak{a}=\mathfrak{g} / \mathfrak{k}$ is Abelian of rank at most $b_{1}(\mathfrak{g})$. This algebra acts on the cohomology of $\mathfrak{k}$ via

$$
\mathrm{A} \cdot[\alpha]=[A\lrcorner d \alpha], \quad \text { for } \mathrm{A} \in \mathfrak{a}=\mathfrak{g} / \mathfrak{k},[\alpha] \in H^{q}(\mathfrak{k}) .
$$

Indeed the above formula defines an action of $\mathfrak{g}$ for which $\mathfrak{k}$ acts trivially. Note that here $d$ is the differential in $\mathfrak{g}$; we will write $d_{0}$ for the differential in $\mathfrak{k}$, so $\alpha \in \operatorname{ker} d_{0}$ in (5.3). Moreover, in our case with $\mathfrak{a}$ Abelian, this action induces the coboundary map $d_{1}$ on the cochains

$$
C^{p}\left(\mathfrak{a}, H^{q}(\mathfrak{k})\right)=\Lambda^{p} \mathfrak{a}^{*} \otimes H^{q}(\mathfrak{k})
$$

via

$$
\left(d_{1} f\right)(\mathrm{a})=\sum_{i=1}^{p+1} f\left(\mathrm{a}_{\wedge i}\right)
$$


for $\mathrm{a} \in \Lambda^{p+1} \mathfrak{a}$.

The Hochschild-Serre spectral sequence [24] has $E_{2}$-page given by the cohomology of the operator $d_{1}$ above:

$$
E_{2}^{p, q}=H^{p}\left(\mathfrak{a}, H^{q}(\mathfrak{k})\right) .
$$

Note that we have

$$
H^{0}\left(\mathfrak{a}, H^{q}(\mathfrak{k})\right)=\left\{b \in H^{q}(\mathfrak{k}): \mathrm{A} \cdot b=0 \text { for all } \mathrm{A} \in \mathfrak{k}\right\}=H^{q}(\mathfrak{k})^{\mathfrak{g}}
$$

the fixed-point set of the action of $G$ on $H^{q}(\mathfrak{k})$. Also note that $E_{2}^{p, q}=0$ for $p>\operatorname{dim} \mathfrak{a}$.

Given the $E_{2}$-page of the spectral sequence, the general theory defines maps $d_{2}: E_{2}^{p, q} \rightarrow E_{2}^{p+2, q-1}$ induced by the exterior derivative $d$ in $\mathfrak{g}$ and sets $E_{3}^{p, q}$ to be the corresponding cohomology group. More generally, $d_{r}: E_{r}^{p, q} \rightarrow$ $E_{r}^{p+r, q-r+1}$ and so the spectral sequence stabilises at level $r=\operatorname{dim} \mathfrak{a}$. So $E_{\infty}^{p, q}=E_{\operatorname{dim} a}^{p, q}$ and one then has

$$
H^{k}(\mathfrak{g}) \cong \bigoplus_{p+q=k} E_{\infty}^{p, q}
$$

Note that if we choose a linear splitting of the exact sequence

$$
0 \rightarrow \mathfrak{k} \rightarrow \mathfrak{g} \rightarrow \mathfrak{a} \rightarrow 0
$$

then the image of $W=\mathfrak{k}^{*}$ in $\mathfrak{g}^{*}$ has $d W \subset \Lambda^{2} W+\mathfrak{a}^{*} \wedge W+\Lambda^{2} \mathfrak{a}^{*}$. In particular, the differential of $\mathfrak{g}$ on $E_{0}^{p, q}$ has components in $E_{0}^{p, q+1}, E_{0}^{p+1, q}$ and $E_{0}^{p+2, q-1}$. Thus this is more general than the spectral sequence of a bicomplex.

We are now ready to state our first characterisation result, the proof of which will be given after some discussion of consequences.

Theorem 5.3. A Lie algebra $\mathfrak{g}$ is $(3,4)$-trivial if and only if $\mathfrak{g}$ is solvable and for any codimension one ideal $\mathfrak{k}$ containing $\mathfrak{g}^{\prime}$ one has $H^{i}(\mathfrak{k})^{\mathfrak{g}}=0$ for $i=2,3$, 4. Here $H^{i}(\mathfrak{k})^{\mathfrak{g}}$ is the part of the cohomology of $\mathfrak{k}$ that is invariant under the action of $\mathfrak{g}$.

This result already gives a number of examples of $(3,4)$-trivial algebras.

Example 5.4. Let $\mathfrak{k}$ be an Abelian algebra $\mathbb{R}^{m}$ of dimension $m$. The differential in $\mathfrak{k}$ is zero and $H^{k}(\mathfrak{k})=\Lambda^{k} \mathfrak{k}^{*}$ for each $k$. Let $T$ be a diagonalisable operator acting by $T\left(\mathrm{~K}_{i}\right)=\lambda_{i} \mathrm{~K}_{i}$, where $\mathrm{K}_{1}, \ldots, \mathrm{K}_{m}$ is a basis of eigenvectors. Write $\kappa_{1}, \ldots, \kappa_{m}$ for the dual basis of $\mathfrak{k}^{*}$. Then $T$ acts on $H^{k}(\mathfrak{k})$ with eigenvalue $\lambda_{i_{1}}+\cdots+\lambda_{i_{k}}$ on $\kappa_{i_{1}} \wedge \cdots \wedge \kappa_{i_{k}}$. We thus have that $H^{i}(\mathfrak{k})^{T}=0$ for $i=2,3,4$ if and only if $\lambda_{i} \neq-\lambda_{j},-\lambda_{j}-\lambda_{k},-\lambda_{j}-\lambda_{k}-\lambda_{\ell}$ whenever $i, j, k, \ell$ are distinct. This then gives a $(3,4)$-trivial algebra $\mathfrak{g}$ defined by

$$
\mathfrak{g}=\mathbb{R A}+\mathfrak{k},
$$




\section{Closed Forms AND MULTI-MOMENT MAPS}

where $\left[\mathrm{A}, \mathrm{K}_{i}\right]=T \mathrm{~K}_{i}=\lambda_{i} \mathrm{~K}_{i}$. Note that if some $\lambda_{i}$ is 0 , then this occurs for only one index $i$ and $\mathfrak{g}$ splits as a product $\mathbb{R}+\mathfrak{h}$, with $T$ acting invertibly on $\mathfrak{h}^{\prime} \cong \mathbb{R}^{m-1}$.

Some concrete $(3,4)$-trivial non-product examples are given as follows. First consider $\mathfrak{k}=\mathbb{R}^{2}$. Then $\mathfrak{g}=(0,12, \mu .13), \mu \neq 0,-1$, is $(3,4)$-trivial. Here the notation means that $\mathfrak{g}^{*}$ has a basis $e_{1}, e_{2}, e_{3}$ with $d e_{1}=0, d e_{2}=$ $e_{1} \wedge e_{2}$ and $d e_{3}=\mu e_{1} \wedge e_{3}$. For a higher-dimensional case with $\mathfrak{k}=\mathbb{R}^{4}$, another example is provided by the algebras $\mathfrak{g}=(0,12,13,14, \mu .15)$ with $\mu \neq 0,-1,-2,-3$. For general $m$, by taking $\lambda_{i}$ strictly positive for each $i$, we obtained $(3,4)$-trivial algebras in all dimensions.

Example 5.5. Suppose $\mathfrak{k}$ is positively graded, meaning that as a vector space $\mathfrak{k}=\mathfrak{k}_{1} \oplus \mathfrak{k}_{2} \oplus \cdots \oplus \mathfrak{k}_{r}$ with $\left[\mathfrak{k}_{i}, \mathfrak{k}_{j}\right] \subset \mathfrak{k}_{i+j}$ for all $i, j$. Such a $\mathfrak{k}$ is necessarily nilpotent. Choosing a linear operator $T$ on $\mathfrak{k}$, we may obtain a Lie algebra $\mathfrak{g}=$ $\mathbb{R A}+\mathfrak{k}$ with $[\mathrm{A}, \mathrm{K}]=T(\mathrm{~K})$ if only if $T\left[\mathrm{~K}_{1}, \mathrm{~K}_{2}\right]=\left[T\left(\mathrm{~K}_{1}\right), \mathrm{K}_{2}\right]+\left[\mathrm{K}_{1}, T\left(\mathrm{~K}_{2}\right)\right]$ for all $\mathrm{K}_{i} \in \mathfrak{k}$. In the positively graded situation we may thus take $T(\mathrm{~K})=j \mathrm{~K}$, for $\mathrm{K} \in \mathfrak{k}_{j}$.

Every nilpotent algebra of dimension at most 6 admits a positive grading (see [29] for concrete gradings), so each of these nilpotent Lie algebras may be realised as the derived algebra of a $(3,4)$-trivial algebra. As a concrete example, the seven-dimensional algebra

$$
(0,12,3.13,4.14+23,5.15+24,6.16+25,7.17+34+26)
$$

obtained from $(0,0,12,13,14,23+15)$ via the grading given by the weights $(1,3,4,5,6,7)$ is $(3,4)$-trivial.

Theorem 5.3 has the following consequence, which completes the characterisation Proposition 5.2 of $(3,4)$-trivial algebras that are direct sums.

Corollary 5.6. Let $\mathfrak{g}$ be a Lie algebra. Write $\mathfrak{k}=\mathfrak{g}^{\prime}$ for the derived algebra and $\mathfrak{a}=\mathfrak{g} / \mathfrak{k}$. Then $\mathfrak{g}$ is $(2,3,4)$-trivial if and only if $\mathfrak{g}$ is solvable, $\mathfrak{a}$ has dimension 1 and $H^{i}(\mathfrak{k})^{\mathfrak{g}}=\{0\}$, for $i=1,2,3,4$.

Proof. By [30] any (2,3)-trivial algebra has codim $\mathfrak{g}^{\prime}=1$ and $H^{i}\left(\mathfrak{g}^{\prime}\right)^{\mathfrak{g}}=\{0\}$, for $i=1,2,3$. Combining this with Theorem 5.3 applied to $\mathfrak{k}=\mathfrak{g}^{\prime}$ gives the result.

Proof (of Theorem 5.3). By Lemma 5.1 we know $\mathfrak{g}$ is solvable, so $\mathfrak{g}^{\prime}$ has codimension at least one. Now with respect to a codimension one ideal $\mathfrak{k}$, the 
$E_{1}$-page of the spectral sequence has row $q$ given by

$$
\begin{array}{c|ccccc}
q+1 & \ldots & & & & \\
q & H^{q}(\mathfrak{k}) \stackrel{d_{1}}{\longrightarrow} \mathfrak{a}^{*} \otimes H^{q}(\mathfrak{k}) & 0 & 0 & \ldots \\
\hline \cdots & 1 & & & \\
\cline { 2 - 5 } & 0 & 1 & 2 & 3 & \ldots
\end{array}
$$

For the $E_{2}$-terms we thus have $E_{2}^{0, q}=\operatorname{ker} d_{1}=H^{q}(\mathfrak{k})^{\mathfrak{g}}$ and $E_{2}^{1, q}=\operatorname{coker} d_{1}$. But $\operatorname{dim} \mathfrak{a}=1$, so $d_{1}$ is a map between vector spaces of the same dimension. This implies coker $d_{1}$ and $\operatorname{ker} d_{1}$ have the same dimension and so we may identify $E_{2}^{1, q}$ with $H^{q}(\mathfrak{k})^{\mathfrak{g}}$, non canonically.

The $E_{2}$-page of the spectral sequence is thus

\begin{tabular}{c|ccccc}
$\vdots$ & $\ldots$ & & & & \\
4 & $H^{4}(\mathfrak{k})^{\mathfrak{g}}$ & $H^{4}(\mathfrak{k})^{\mathfrak{g}}$ & 0 & 0 & $\ldots$ \\
3 & $H^{3}(\mathfrak{k})^{\mathfrak{g}}$ & $H^{3}(\mathfrak{k})^{\mathfrak{g}}$ & 0 & 0 & $\ldots$ \\
2 & $H^{2}(\mathfrak{k})^{\mathfrak{g}}$ & $H^{2}(\mathfrak{k})^{\mathfrak{g}}$ & 0 & 0 & $\ldots$ \\
1 & $H^{1}(\mathfrak{k})^{\mathfrak{g}}$ & $H^{1}(\mathfrak{k})^{\mathfrak{g}}$ & 0 & 0 & $\ldots$ \\
0 & $\mathbb{R}$ & $\mathbb{R}$ & 0 & 0 & $\ldots$ \\
\hline & 0 & 1 & 2 & 3 & $\ldots$
\end{tabular}

The spectral sequence degenerates at the $E_{2}$-term and we conclude that

$$
H^{3}(\mathfrak{g}) \cong H^{3}(\mathfrak{k})^{\mathfrak{g}}+H^{2}(\mathfrak{k})^{\mathfrak{g}}, \quad H^{4}(\mathfrak{g}) \cong H^{4}(\mathfrak{k})^{\mathfrak{g}}+H^{3}(\mathfrak{k})^{\mathfrak{g}},
$$

from which the result follows.

In the case when $\mathfrak{n}$ is a nilpotent Lie algebra, one may use the above spectral sequence to prove Dixmier's result [14] that $b_{k}(\mathfrak{n}) \geqslant 2$ for each $0<k<\operatorname{dim} \mathfrak{n}$, since in this situation $\mathfrak{g}=\mathfrak{n}$ acts nilpotently on $H^{q}(\mathfrak{k})$ and so $H^{q}(\mathfrak{k})^{\mathfrak{g}}$ is non-zero if $H^{q}(\mathfrak{k})$ is non-zero. A refinement of the above argument was also used by Cairns and Jessup [10] to improve Dixmier's bounds.

Proposition 5.7. A Lie algebra $\mathfrak{g}$ with derived algebra $\mathfrak{g}^{\prime}$ of codimension at least two is $(3,4)$-trivial if and only if for each ideal $\mathfrak{k}$ of $\mathfrak{g}$ containing $\mathfrak{g}^{\prime}$ one has $H^{i}(\mathfrak{k})^{\mathfrak{g}}=0$ for $i=1,2,3,4$.

The reason for giving this result in addition to Theorem 5.3 is that we will see later that we can often assume that $\operatorname{codim} \mathfrak{g}^{\prime} \leqslant 2$ and therefore take $\mathfrak{k}=\mathfrak{g}^{\prime}$ to be nilpotent. 


\section{Closed Forms AND MULTI-MOMENT MAPS}

Example 5.8. Suppose $\mathfrak{k}$ is Abelian as in Example 5.4. We may now consider two commuting diagonalisable operators $T_{1}$ and $T_{2}$ acting on $\mathfrak{k}$. Then $\mathfrak{k}$ splits as a direct sum of common eigenspaces of $T_{1}$ and $T_{2}$. We wish to consider when the Lie algebra given by

$$
\begin{gathered}
\mathfrak{g}=\left\langle\mathrm{A}_{1}, \mathrm{~A}_{2}\right\rangle+\mathfrak{k}, \\
{\left[s \mathrm{~A}_{1}+t \mathrm{~A}_{2}, \mathrm{~K}\right]=\left(s T_{1}+t T_{2}\right)(\mathrm{K}), \quad \text { for } s, t \in \mathbb{R}, \mathrm{K} \in \mathfrak{k},}
\end{gathered}
$$

is $(3,4)$-trivial.

The cohomology conditions of Proposition 5.7 are satisfied if $T_{1}$ and $T_{2}$ have no common 0-eigenspace on $\Lambda^{i} \mathfrak{k}^{*}$ for $i=1,2,3,4$. This is a vanishing condition on finitely many linear combinations of the eigenvalues of $T_{1}$ and of $T_{2}$. When it is satisfied we may thus find a generic linear combination $T=s T_{1}+t T_{2}$ such that the action of $T$ on these $\Lambda^{i} \mathfrak{k}^{*}$ has trivial kernel. Taking $T_{1}=T$, we are now free to have $T_{2}$ any linear transformation of $\mathfrak{k}$ that commutes with $T_{1}$, and need not assume that $T_{2}$ is diagonalisable.

Thus for example when $\mathfrak{k} \cong \mathbb{R}^{2}$, we see that the algebra

$$
(0,0,13+24,14)
$$

is $(3,4)$-trivial.

Example 5.9. If $\mathfrak{k}$ is positively graded, as in Example 5.5, we may take $T_{1}=T$ as in that example and let $A_{2}$ be any derivation $T_{2}$ of $\mathfrak{k}$ commuting with $T_{1}$. Then (5.4) defines a $(3,4)$-trivial algebra. A simple example is obtained by taking $\mathfrak{k}$ to be the Heisenberg algebra $(0,0,12)$. This has a positive grading with weights $(1,1,2)$. A second derivation $T_{2}$ acts on $\mathfrak{k}^{*}$ by $e_{1} \mapsto e_{2}, e_{2} \mapsto$ $-e_{1}, e_{3} \mapsto 0$ and this commutes with $T_{1}$. We thus conclude that

$$
(0,0,13+24,14-23,2.15)
$$

is $(3,4)$-trivial. Another choice for $T_{2}$ is the nilpotent transformation $e_{1} \mapsto$ $e_{2}, e_{2} \mapsto 0, e_{3} \mapsto 0$ of $\mathfrak{k}^{*}$, which gives the (3,4)-trivial algebra

$$
(0,0,13+24,14,2.15)
$$

Proof (of Proposition 5.7). This time $\mathfrak{a}^{*} \cong \mathbb{R}^{2}$ and $\Lambda^{2} \mathfrak{a}^{*} \cong \mathbb{R}$, so the $E_{1}$-page of the spectral sequence has row $q$ isomorphic to

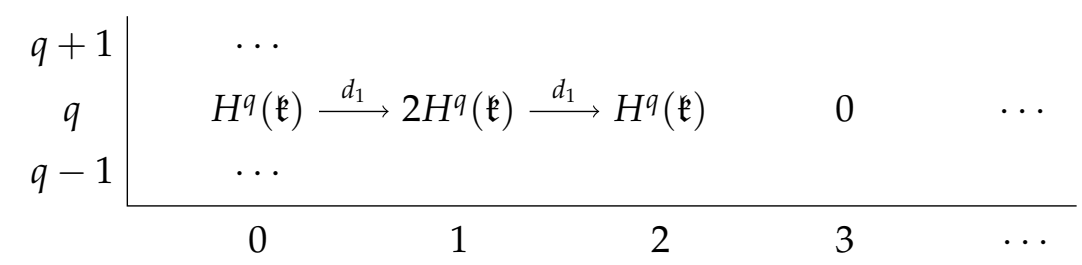


Since the maps $d_{1}$ on the bottom row $q=0$ are zero, the relevant part of the $E_{2}$-page is

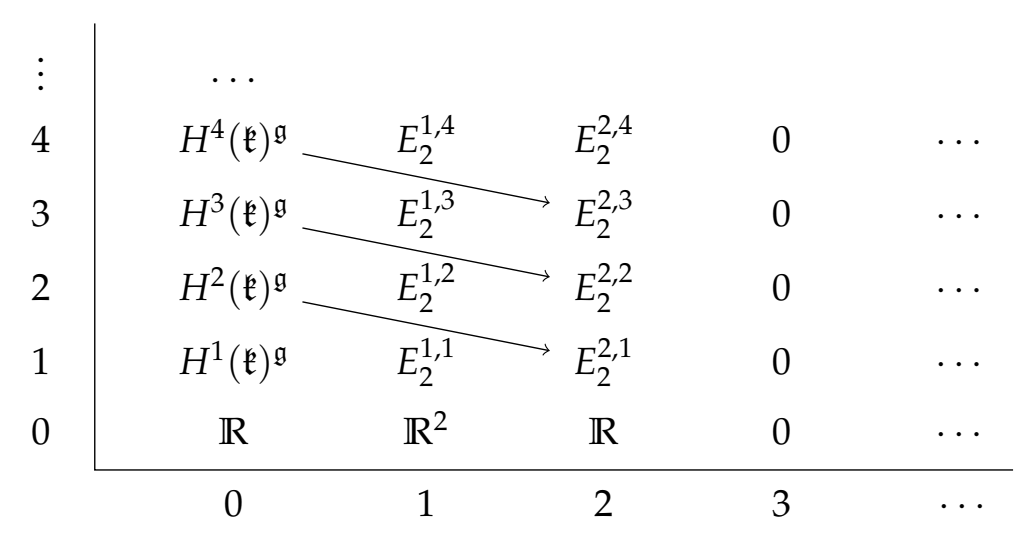

The maps $d_{2}$ from and to the middle column $E_{2}^{1, p}$ are zero, and the spectral sequence degenerates at the $E_{3}$-level, so

$$
\begin{aligned}
& H^{3}(\mathfrak{g}) \cong E_{3}^{0,3}+E_{2}^{1,2}+E_{3}^{2,1} \\
& H^{4}(\mathfrak{g}) \cong E_{3}^{0,4}+E_{2}^{1,3}+E_{3}^{2,2} .
\end{aligned}
$$

Thus $b_{3}(\mathfrak{g})=0=b_{4}(\mathfrak{g})$ implies that $E_{2}^{1,2}=0=E_{2}^{1,3}$. This says that the middle cohomologies of the $E_{1}$-page on rows $q=2$ and $q=3$ are zero. However, for a sequence $V \stackrel{\alpha}{\rightarrow} 2 V \stackrel{\beta}{\rightarrow} V$, vanishing of the middle cohomo$\operatorname{logy}$ implies that $\alpha$ is injective and $\beta$ is surjective. Thus the cohomologies at each end of these rows are also zero. In particular,

$$
H^{2}(\mathfrak{k})^{\mathfrak{g}}=0 \quad \text { and } \quad H^{3}(\mathfrak{k})^{\mathfrak{g}}=0 .
$$

The $E_{2}$-page is now

\begin{tabular}{c|ccccc}
$\vdots$ & $\ldots$ & & & & \\
4 & $H^{4}(\mathfrak{k})^{\mathfrak{g}}$ & $E_{2}^{1,4}$ & $E_{2}^{2,4}$ & 0 & $\ldots$ \\
3 & 0 & 0 & 0 & 0 & $\ldots$ \\
2 & 0 & 0 & 0 & 0 & $\ldots$ \\
1 & $H^{1}(\mathfrak{k})^{\mathfrak{g}}$ & $E_{2}^{1,1}$ & $E_{2}^{2,1}$ & 0 & $\ldots$ \\
0 & $\mathbb{R}$ & $\mathbb{R}^{2}$ & $\mathbb{R}$ & 0 & $\ldots$ \\
\hline & 0 & 1 & 2 & 3 & $\ldots$
\end{tabular}




\section{Closed Forms AND MULTI-MOMENT MAPS}

all the $d_{2}$ maps marked in the first picture of the $E_{2}$-page are zero and

$$
H^{3}(\mathfrak{g}) \cong E_{2}^{2,1}, \quad H^{4}(\mathfrak{g}) \cong H^{4}(\mathfrak{k})^{\mathfrak{g}}
$$

Thus (3,4)-trivial implies

$$
H^{4}(\mathfrak{k})^{\mathfrak{g}}=0
$$

To prove the necessity of the cohomology conditions it only remains to show that $E_{2}^{2,1}=0$ if and only if $H^{1}(\mathfrak{k})^{\mathfrak{g}}=0$. In fact to get sufficiency we will show that $H^{q}(\mathfrak{k})^{\mathfrak{g}}=0$ is equivalent to $E_{2}^{2, q}=0$.

Write $V=H^{q}(\mathfrak{k}) \otimes \mathbb{C}=H^{q}(\mathfrak{k})_{\mathbb{C}}$ and let $\mathrm{A}, \mathrm{B}$ be a basis for $\mathfrak{a}$. The space $\left(E_{2}^{2, q}\right)_{\mathrm{C}}$ is the cokernel of $d_{1}: V \times V \rightarrow V$ with $d_{1}\left(f_{1}, f_{2}\right)=A f_{2}-B f_{1}$, where $A, B$ are the linear operators of the action of $A$ and $B$ on $V$. Thus $\left(E_{2}^{2, q}\right)_{\mathrm{C}}$ is $V /(\operatorname{im} A+\operatorname{im} B)$, whereas $H^{q}(\mathfrak{k})_{\mathbb{C}}^{\mathfrak{g}}$ is $\operatorname{ker} A \cap \operatorname{ker} B$.

Now $A$ and $B$ commute, so each preserves the generalised eigenspaces of the other. Decompose $V$ as a direct sum of the common generalised eigenspaces $V=\bigoplus E(\lambda)$, where $E\left(\lambda_{1}, \lambda_{2}\right)=E_{A}\left(\lambda_{1}\right) \cap E_{B}\left(\lambda_{2}\right)=\operatorname{ker}((A-$ $\left.\left.\lambda_{1}\right)^{n}\right) \cap \operatorname{ker}\left(\left(B-\lambda_{2}\right)^{n}\right), n=\operatorname{dim} V$. We see that $A$ or $B$ is invertible on each space $E(\lambda)$ with $\lambda \neq(0,0)$. Thus $\operatorname{ker} A \cap \operatorname{ker} B$ is a subspace of $E(0)$ and $V /(\operatorname{im} A+\operatorname{im} B)$ is a quotient space of $E(0)$.

On $E(0)$, the operators $A$ and $B$ are nilpotent and commute. They thus generate a nilpotent Lie algebra and by Engel's Theorem, there is a basis $v_{1}, \ldots, v_{m}$ for $E(0)$ such that $A$ and $B$ are upper triangular. In particular $\operatorname{im} A+\operatorname{im} B \leqslant\left\langle v_{1}, \ldots, v_{m-1}\right\rangle$ and $v_{1} \in \operatorname{ker} A \cap \operatorname{ker} B$. Thus $V /(\operatorname{im} A+\operatorname{im} B)$ or $\operatorname{ker} A \cap \operatorname{ker} B$ is zero, if and only if $E(0)$ is zero. This proves that $H^{q}(\mathfrak{k})^{\mathfrak{g}}=$ 0 if and only if $E_{2}^{2, q}=0$.

We thus have that the given cohomology conditions are necessary. To see that they are sufficient, note that the vanishing of $H^{q}(\mathfrak{k})^{\mathfrak{g}}$ implies that the sequence on the $q$-row of the $E_{1}$-page has the form $V \stackrel{\alpha}{\rightarrow} 2 V \stackrel{\beta}{\rightarrow} V$ with $\alpha$ injective and $\beta$ surjective. It follows that the middle cohomology is also zero and that $E_{2}^{p, q}$ is zero for all $1 \leqslant p \leqslant 4$. This implies that $b_{3}(\mathfrak{g})=0=b_{4}(\mathfrak{g})$ as required.

Theorem 5.3 and Proposition 5.7 give a full structural description of the $(3,4)$-trivial algebras with $\mathfrak{g}^{\prime}$ of codimension at most two. The next result gives a couple of conditions under which this codimension assumption is guaranteed.

Proposition 5.10. A Lie algebra $\mathfrak{g}$ with $b_{3}(\mathfrak{g})=0$ has derived algebra $\mathfrak{k}=\mathfrak{g}^{\prime}$ of codimension at most two if either

(i) $\mathfrak{g}$ is split, so $\mathfrak{g}$ is the semi-direct product of $\mathfrak{a}=\mathfrak{g} / \mathfrak{k}$ and $\mathfrak{k}$, or

(ii) $H^{1}(\mathfrak{k})^{\mathfrak{g}}=\{0\}=H^{2}(\mathfrak{k})^{\mathfrak{g}}$. 
Proof. When $\mathfrak{g}$ is split, we have $\mathfrak{g}=\mathfrak{a}+\mathfrak{k},[\mathfrak{a}, \mathfrak{a}]=0$, and so the differential $d$ of $\mathfrak{g}$ satisfies $d \mathfrak{a}^{*}=0$ and $d \mathfrak{k}^{*} \subset \Lambda^{2} \mathfrak{k}^{*}+\mathfrak{a}^{*} \wedge \mathfrak{k}^{*}$. In particular, no element of $\Lambda^{k} \mathfrak{a}^{*}$ is exact, and there is an injection $\Lambda^{k} \mathfrak{a}^{*} \rightarrow H^{k}(\mathfrak{g})$ for each $k$. Thus $b_{3}(\mathfrak{g})=0$ implies $\Lambda^{3} \mathfrak{a}^{*}=0$ and $\mathfrak{a}$ has dimension at most two, as required.

For the second case of the Proposition, we consider the spectral sequence. Choose a basis $A_{1}, \ldots, A_{r}$ for $\mathfrak{a}$. The proof of Proposition 5.7 shows that $H^{q}(\mathfrak{k})^{\mathfrak{g}}=\{0\}$ is equivalent to common generalised eigenspace $E(0)=\bigcap_{i=1}^{r} \operatorname{ker}\left(A_{i}^{n}\right)$ of the induced operators $A_{i}$ on $H^{q}(\mathfrak{k})$ being zero. This implies that some linear combination $\mathrm{A}$ of the $\mathrm{A}_{i}$ acts invertibly on $H^{q}(\mathfrak{k})$. We may thus choose our basis $\mathrm{A}_{i}$ so that $A_{1}$ acts invertibly on the given $H^{q}(\mathfrak{k})$.

Now consider the cohomology of

$$
H^{q}(\mathfrak{k}) \stackrel{d_{1}}{\longrightarrow} \mathfrak{a}^{*} \otimes H^{q}(\mathfrak{k}) \stackrel{d_{1}}{\longrightarrow} \Lambda^{2} \mathfrak{a}^{*} \otimes H^{q}(\mathfrak{k})
$$

The first map is given by $v \mapsto\left(A_{1} v, \ldots, A_{r} v\right)$, the second by $\left(f_{1}, \ldots, f_{r}\right) \mapsto$ $\left(A_{i} f_{j}-A_{j} f_{i}\right)$. For $\mathbf{f}=\left(f_{1}, \ldots, f_{r}\right) \in \operatorname{ker} d_{1} \subset \mathfrak{a}^{*} \otimes H^{q}(\mathfrak{k})$, we may write $v=A_{1}^{-1} f_{1}$. Then $A_{1} f_{j}-A_{j} f_{1}=0$, implies that $f_{j}=A_{1}^{-1}\left(A_{j} f_{1}\right)=A_{j} v$ and that $\mathbf{f} \in \operatorname{im} d_{1}$. Thus the $d_{1}$-cohomology vanishes at the second step.

Under the hypotheses of the Proposition, the $E_{2}$-page of the spectral sequence for $\mathfrak{g}$ with respect the derived algebra $\mathfrak{k}$ is now

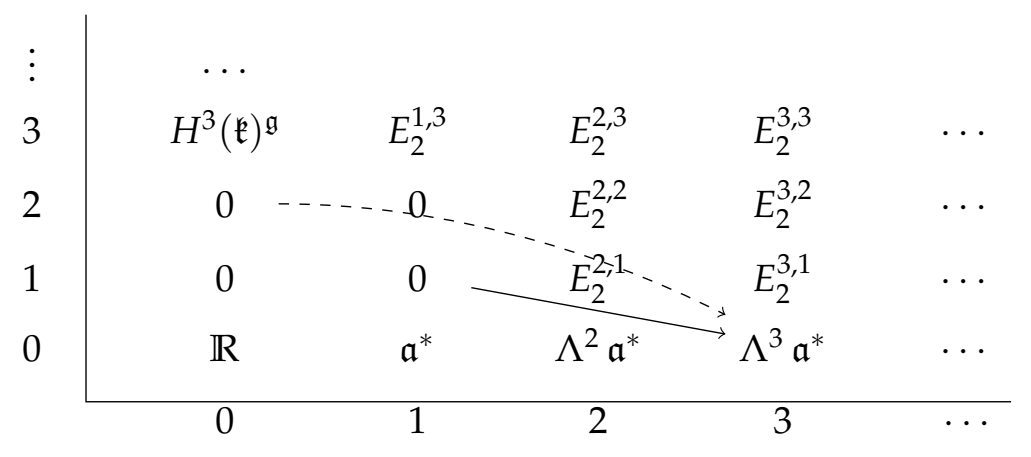

with the solid arrow to $\Lambda^{3} \mathfrak{a}^{*}$ representing $d_{2}$ and the dashed arrow indicating $d_{3}$. This gives that $\Lambda^{3} \mathfrak{a}^{*}=E_{2}^{3,0}=E_{3}^{3,0}=E_{\infty}^{3,0}$ is a summand of $H^{3}(\mathfrak{g})$. Hence $b_{3}(\mathfrak{g}) \geqslant \operatorname{dim} \Lambda^{3} \mathfrak{a}^{*}$ and $b_{3}(\mathfrak{g})=0$ implies that $\operatorname{dim} \mathfrak{a} \leqslant 2$, as required.

Remark 5.11. Note that the condition $H^{1}(\mathfrak{k})^{\mathfrak{g}}=\{0\}$ does not appear in Theorem 5.3. However, when $\mathfrak{k}=\mathfrak{g}^{\prime}$ is of codimension one, this space is automatically zero. Indeed, suppose that $\mathfrak{g}=\mathbb{R A}+\mathfrak{k}$ with $\mathfrak{k}$ an ideal. A non-zero element of $H^{1}(\mathfrak{k})^{\mathfrak{g}}$ is an element $\gamma \in \mathfrak{k}^{*}$, such that $d_{0} \gamma=0$ and A $\cdot \gamma=0$. The first condition implies that $\gamma$ annihilates $\mathfrak{k}^{\prime}$, the second says that $\gamma([\mathrm{A}, \mathrm{K}])=0$ for each $\mathrm{K} \in \mathfrak{k}$. Thus $\gamma$ annihilates $\mathfrak{g}^{\prime}$, but so does $\mathfrak{a}^{*}$, so $\mathfrak{g}^{\prime}$ is at least codimension two. 


\section{Closed Forms AND MULTI-MOMENT MAPS}

The Betti numbers of Lie algebras of dimension at most 6 are given in Freibert and Schulte-Hengesbach [16, 17]. One may thus use their tables to read off which algebras of dimension 4,5 or 6 are $(3,4)$-trivial. Table 5.1 summarises the resulting sets of Betti numbers that occur. The tables confirm that $\mathfrak{g}^{\prime}$ is of codimension at most two in these cases. When the codimension equals two, i.e., $b_{1}(\mathfrak{g})=2$, one sees that $b_{n}(\mathfrak{g})=0$. In other words, these examples are not unimodular. The next example shows that this is no longer true in higher dimensions.

\begin{tabular}{cc}
\hline$n=\operatorname{dim} \mathfrak{g}$ & $\left(b_{1}, \ldots, b_{n}\right)$ \\
\hline 4 & $(1,0,0,0),(2,1,0,0)$ \\
5 & $(1,0,0,0,0),(2,1,0,0,0)$ \\
6 & $(1,0,0,0,0,0),(1,0,0,0,1,1),(2,1,0,0,0,0)$ \\
\hline
\end{tabular}

Table 5.1. The sets of Betti numbers of the $(3,4)$-trivial Lie algebras $\mathfrak{g}$ appearing in the low-dimensional classifications of [16, 17].

Example 5.12. Consider an $(m+2)$-dimensional Lie algebra of the form as in Example 5.8 with $T_{1}$ and $T_{2}$ commuting and diagonalisable. The proof of Proposition 5.7 shows that we may assume that $T_{1}$ is invertible. The action of $T_{i}$ on $H^{m}(\mathfrak{k})$ is simply the trace of the action $\mathfrak{k}^{*}$. When $m \geqslant 5$, we may ensure that the cohomology conditions of Proposition 5.7 are satisfied and that each $T_{i}$ acts trivially on $H^{m}(\mathfrak{k})$, for example by giving both $T_{i}$ strictly positive eigenvalues on some $(m-1)$-dimensional subspace of $\mathfrak{k}$ and requiring $T_{i}$ to be trace-free. Now this trace-free condition ensures that the maps $d_{1}: E_{1}^{p, m} \rightarrow E_{1}^{p+1, m}$ of the Hochschild-Serre spectral sequence for $\mathfrak{g}$ with respect to the ideal $\mathfrak{k}$ are identically zero, so $H^{n}(\mathfrak{g})=E_{2}^{2, m}=$ $E_{1}^{2, m}=H^{m}(\mathfrak{k})=\mathbb{R}$, showing that $\mathfrak{g}$ is unimodular. A concrete non-product unimodular $(3,4)$-trivial algebra of dimension $n=m+2 \geqslant 7$ with $b_{1}=2$ is given by

$$
(0,0,13+23,14,15, \ldots,(1-m) \cdot 1 n-2 n) .
$$

\section{References}

[1] John C. Baez, Alexander E. Hoffnung and Christopher L. Rogers. 'Categorified symplectic geometry and the classical string'. In: Comm. Math. Phys. 293.3 (2010), pp. 701-725. ISSN: 0010-3616. DOr: 10.1007/s00220-009-0951-9. URL: http://dx.doi.org/10.1007/s00220-009-0951-9

[2] John C. Baez and Christopher L. Rogers. 'Categorified symplectic geometry and the string Lie 2-algebra'. In: Homology, Homotopy Appl. 12.1 (2010), pp. 221236. IsSN: 1532-0073. uRL:http://projecteuclid.org/getRecord?id=euclid.hha/1296223828.

[3] C. Bär. 'Real Killing spinors and holonomy'. In: Comm. Math. Phys. 154 (1993), pp. 509-521. 


\section{Thomas Bruun Madsen \& Andrew Swann}

[4] H. Baum, Th. Friedrich, R. Grunewald and I. Kath. Twistors and Killing spinors on Riemannian manifolds. Stuttgart, Leipzig: B. G. Teubner Verlagsgesellschaft, 1991.

[5] M. Berger. 'Sur les groupes d'holonomie des variétés à connexion affine et des variétés riemanniennes'. In: Bull. Soc. Math. France 83 (1955), pp. 279-330.

[6] A. L. Besse. Einstein manifolds. Vol. 10. Ergebnisse der Mathematik und ihrer Grenzgebiete, 3. Folge. Berlin, Heidelberg and New York: Springer, 1987.

[7] E. Bonan. 'Sur des variétés riemanniennes à groupe d'holonmie $G_{2}$ ou $\operatorname{Spin}(7)$ '. In: C. R. Acad. Sci. Paris 262 (1966), pp. 127-129.

[8] R. L. Bryant. 'Metrics with exceptional holonomy'. In: Ann. of Math. 126 (1987), pp. 525-576.

[9] R. L. Bryant and S. M. Salamon. 'On the construction of some complete metrics with exceptional holonomy'. In: Duke Math. J. 58 (1989), pp. 829-850.

[10] Grant Cairns and Barry Jessup. 'New bounds on the Betti numbers of nilpotent Lie algebras'. In: Comm. Algebra 25.2 (1997), pp. 415-430. IssN: 0092-7872. DOI: 10.1080/00927879708825863, uRL:http://dx.doi.org/10.1080/00927879708825863

[11] J. F. Carinena, J. Clemente-Gallardo and G. Marmo. Reduction Procedures in Classical and Quantum Mechanics. Sept. 2007. arXiv:0709.2366v1 [math-ph].

[12] J. F. Cariñena, M. Crampin and L. A. Ibort. 'On the multisymplectic formalism for first order field theories'. In: Differential Geom. Appl. 1.4 (1991), pp. 345-374. ISSN: 0926-2245. DOI: 10.1016/0926-2245(91) 90013-Y. URL: http://dx.doi.org/10.1016/092

[13] Diego Conti and Simon Salamon. 'Generalized Killing spinors in dimension 5'. In: Trans. Amer. Math. Soc. 359.11 (2007), pp. 5319-5343. IssN: 0002-9947.

[14] J. Dixmier. 'Cohomologie des algèbres de Lie nilpotentes'. In: Acta Sci. Math. Szeged 16 (1955), pp. 246-250.

[15] M. Fernández and A. Gray. 'Riemannian manifolds with structure group $G_{2}$ '. In: Ann. Mat. Pura Appl. (4) 132 (1982), 19-45 (1983). IssN: 0003-4622.

[16] Marco Freibert and Fabian Schulte-Hengesbach. Half-flat structures on decomposable Lie groups. Transform. Groups (to appear). Dec. 2010. arXiv:1110.1512 [math.DG]

[17] Marco Freibert and Fabian Schulte-Hengesbach. Half-flat structures on indecomposable Lie groups. Oct. 2011. arXiv:1110.1512 [math.DG].

[18] T. Friedrich, I. Kath, A. Moroianu and U. Semmelmann. 'On nearly parallel G ${ }_{2}$-structures'. In: J. Geom. Phys. 23.3-4 (1997), pp. 259-286. ISSN: 0393-0440.

[19] Mark J. Gotay, James Isenberg, Jerrold E. Marsden and Richard Montgomery. Momentum Maps and Classical Relativistic Fields. Part I: Covariant Field Theory. Jan. 1998. arXiv physics/9801019 [math-ph].

[20] U. Gran, J. Gutowski and G. Papadopoulos. 'IIB black hole horizons with five-form flux and KT geometry'. In: J. High Energy Phys. 5 (2011), p. 050.

[21] A. Gray. 'A note on manifolds whose holonomy group is a subgroup of $S p(n) \cdot S p(1)^{\prime}$. In: Michigan Math. J. 16 (1969), pp. 125-128. In: 17 (1970), p. 409.

[22] A. Gray. 'Weak holonomy groups'. In: Math. Z. 123 (1971), pp. 290-300.

[23] N. J. Hitchin. 'Stable forms and special metrics'. In: Global differential geometry: the mathematical legacy of Alfred Gray (Bilbao, 2000). Vol. 288. Contemp. Math. Providence, RI: Amer. Math. Soc., 2001, pp. 70-89.

[24] G. Hochschild and J.-P. Serre. 'Cohomology of Lie algebras'. In: Ann. of Math. (2) 57 (1953), pp. 591-603. Issn: 0003-486X.

[25] D. Joyce. 'Compact Riemannian 8-manifolds with holonomy Spin(7)'. In: Invent. Math. 123 (1996), pp. 507-552. 


\section{Closed Forms AND MULTI-MOMENT MAPS}

[26] S. Karigiannis. 'Deformations of $G_{2}$ and Spin(7) structures'. In: Canad. J. Math. 57.5 (2005), pp. 1012-1055. ISSN: 0008-414X.

[27] T. B. Madsen. 'Spin(7)-manifolds with three-torus symmetry'. In: J. Geom. Phys. 61.11 (2011), pp. 2285-2292. IssN: 0393-0440. DoI: doi:10.1016/j .geomphys. 2011.07.008

[28] T. B. Madsen. 'Torsion geometry and scalar functions'. PhD thesis. University of Southern Denmark, 2011.

[29] T. B. Madsen and A. F. Swann. 'Homogeneous spaces, multi-moment maps and $(2,3)$-trivial algebras'. In: Proceedings of the XIXth International Fall Workshop on Geometry and Physics, Porto, September 6-9, 2010. Vol. 1360. AIP Conference Proceedings. American Institute of Physics, 2011, pp. 51-62. arXiv 1012.0402 [math.DG].

[30] T. B. Madsen and A. F. Swann. Multi-moment maps. IMADA preprint 2010, CP3-ORIGINS: 2010-53. Dec. 2010. arXiv 1012.2048 [math.DG].

[31] W. S. Massey. 'Cross products of vectors in higher-dimensional Euclidean spaces'. In: Amer. Math. Monthly 90.10 (1983), pp. 697-701. IssN: 0002-9890. DOI: $10.2307 / 2323537$. URL: http://dx.doi.org/10.2307/2323537

[32] Karl-Hermann Neeb and Cornelia Vizman. 'An abstract setting for Hamiltonian actions'. In: Monatsh. Math. 159.3 (2010), pp. 261-288.

[33] Y. S. Poon and A. F. Swann. 'Superconformal symmetry and hyperKähler manifolds with torsion'. In: Commun. Math. Phys. 241.1 (2003), pp. 177-189.

[34] Frank Reidegeld. 'Spaces admitting homogeneous $G_{2}$-structures'. In: Differential Geom. Appl. 28.3 (2010), pp. 301-312. ISSN: 0926-2245. Dor: $10.1016 /$ j.difgeo.2009.10.013 URL: http://dx.doi.org/10.1016/j.difgeo.2009.10.013.

[35] Frank Reidegeld. 'Special cohomogeneity-one metrics with $Q^{1,1,1}$ or $M^{1,1,0}$ as the principal orbit'. In: J. Geom. Phys. 60.9 (2010), pp. 1069-1088. IssN: 03930440. DoI: $10.1016 / j$.geomphys. 2010.03.006 URL: http://dx.doi.org/10.1016/j.geomphys. 2

[36] A. F. Swann. 'Aspects symplectiques de la géométrie quaternionique'. In: C. R. Acad. Sci. Paris 308 (1989), pp. 225-228.

[37] A. F. Swann. 'HyperKähler and quaternionic Kähler geometry'. In: Math. Ann. 289 (1991), pp. 421-450.

[38] George W. Whitehead. 'Note on cross-sections in Stiefel manifolds'. In: Comment. Math. Helv. 37 (1962/1963), pp. 239-240. IssN: 0010-2571.

[39] F. Witt. 'Special metrics and triality'. In: Adv. Math. 219.6 (2008), pp. 19722005. IssN: 0001-8708.

[40] F. Witt. 'Special metric structures and closed forms'. eprint arXiv:math/0502443v2 [math.DG]. $\mathrm{PhD}$ thesis. University of Oxford, 2004.

[41] J. A. Wolf. 'The geometry and topology of isotropy irreducible homogeneous spaces'. In: Acta Math. 120 (1968), pp. 59-148. In: 152.1-2 (1984), pp. 141-142.

\section{T.B.Madsen \& A.F.Swann}

Department of Mathematical Sciences, University of Aarhus, Ny Munkegade 118, Bldg 1530, DK-8000 Aarhus C, Denmark.

and

$\mathrm{CP}^{3}$-Origins, Centre of Excellence for Particle Physics Phenomenology, University of Southern Denmark, Campusvej 55, DK-5230 Odense M, Denmark. 
Thomas Bruun Madsen \& Andrew Swann

E-mail: tbmadsen@imf .au.dk, swann@imf .au.dk 